\title{
ANALYSIS OF TRANSIENT ACOUSTIC-ELASTIC INTERACTION IN AN UNBOUNDED STRUCTURE
}

\author{
YIXIAN GAO*, PEIJUN $\mathrm{LI}^{\dagger}$, AND BO ZHANG
}

\begin{abstract}
Consider the wave propagation in a two-layered medium consisting of a homogeneous compressible air or fluid on top of a homogeneous isotropic elastic solid. The interface between the two layers is assumed to be an unbounded rough surface. This paper concerns the time-domain analysis of such an acoustic-elastic interaction problem in an unbounded structure in three dimensions. Using an exact transparent boundary condition and suitable interface conditions, we study an initialboundary value problem for the coupling of the Helmholtz equation and the Navier equation. The well-posedness and stability are established for the reduced problem. Our proof is based on the method of energy, the Lax-Milgram lemma, and the inversion theorem of the Laplace transform. Moreover, a priori estimates with explicit dependence on the time are achieved for the quantities of acoustic pressure and elastic displacement by taking special test functions for the time-domain variational problem.
\end{abstract}

Key words. Acoustic wave equation, elastic wave equation, unbounded rough surface, time domain, stability, priori estimates

AMS subject classifications. 78A46, 65C 30

1. Introduction. Consider a two-layered medium which consists of a homogeneous compressible air or fluid on top of a homogeneous isotropic elastic solid. The interface between air/fluid and solid is assumed to be an unbounded rough surface. An unbounded rough surface refers to a non-local perturbation of an infinite plane surface such that the whole surface lies within a finite distance of the original plane. As a source located in the solid, the external force generates an elastic wave, which propagates towards the interface and further excites an acoustic wave in the air/fluid. This process leads to an air/fluid-solid interaction problem with an unbounded interface separating the acoustic and elastic waves which are coupled on the interface through two continuity conditions. The first kinematic interface condition is imposed to ensure that the normal velocity of the air/fluid on one side of the boundary matches the accelerated velocity of the solid on another side. The second one is the dynamic condition which results from the balance of forces on two sides of the interface. The model problem describes the seismic wave propagation in the air/fluid-solid medium due to the excitation of an earthquake source which is located in the crust between the lithosphere and the mantle of the Earth. The goal of this paper is to carry the mathematical analysis of the time-domain acoustic-elastic scattering problem in such an unbounded structure in three dimensions.

This problem falls into the class of unbounded rough surface scattering problems, which have been of great interest to physicists, engineers, and applied mathematicians for many years due to their wide range of applications in optics, acoustics, radio-wave propagation, seismology, and radar techniques [1, 11, 30, 35, 38. The elastic wave

*School of Mathematics and Statistics, Center for Mathematics and Interdisciplinary Sciences, Northeast Normal University, Changchun, Jilin 130024, China. This author's research was supported in part by NSFC grant 11571065 and Jilin Science and Technology Development Project. (gaoyx643@nenu.edu.cn)

${ }^{\dagger}$ Department of Mathematics, Purdue University, West Lafayette, Indiana 47907, USA. This author's research was supported in part by the NSF grant DMS-1151308. (lipeijun@math.purdue.edu)

${ }^{\ddagger}$ LSEC and Institute of Applied Mathematics, AMSS, Chinese Academy of Sciences, Beijing 100190, China. This author's research was partially supported by the NNSFC grants 61379093 and 91430102. (b.zhang@amt.ac.cn) 
scattering by unbounded interfaces has many important applications in geophysics and seismology. For instance, the problem of elastic pulse transmission and reflection through the Earth is fundamental to the investigation of earthquakes and the utility of controlled explosions in search for oil and ore bodies [14,15,32. The unbounded rough surface scattering problems are quite challenging due to the unbounded surfaces. The usual Sommerfeld (for acoustic waves) or Silver-Müller (for electromagnetic waves) radiation condition is not valid any more [2 40. The Fredholm alternative theorem is not applicable either due to the lack of compactness result. For the time-harmonic problems, we refer to $[3,5,23,25]$ for some mathematical studies on the two-dimensional Helmholtz equation and [17,27,28 for the three-dimensional Maxwell equations. The time-domain scattering problems have recently attracted considerable attention due to their capability of capturing wide-band signals and modeling more general material and nonlinearity $6,22,24,31,37$, which motivates us to tune our focus from seeking the best possible conditions for those physical parameters to the time-domain problem. Comparing with the time-harmonic problems, the time-domain problems are less studied due to the additional challenge of the temporal dependence. The analysis can be found in [7,36 for the time-domain acoustic and electromagnetic obstacle scattering problems. We refer to [26] and [16] for the analysis of the time-dependent electromagnetic scattering from an open cavity and a periodic structure, respectively.

The acoustic-elastic interaction problems have received much attention in both the mathematical and engineering communities $9,10,18,20,29$. . There are also some numerical studies on the inverse problems arising from the fluid-solid interaction such as reconstruction of surfaces of periodic structures or obstacles [21,39. Many approaches have been attempted to solve numerically the time-domain problems such as coupling of boundary element and finite element with different time quadratures [12, 13, 33. However, the rigorous mathematical study is still open at present.

In this work, we intend to answer the mathematical questions on well-posedness and stability of the time-domain acoustic-elastic interaction problem in an unbounded structure. The problem is reformulated as an initial-boundary value problem by adopting an exact transparent boundary condition (TBC). Using the Laplace transform and energy method, we show that the reduced variational problem has a unique weak solution in the frequency domain. Meanwhile, we obtain the stability estimate to show the existence of the solution in the time-domain. In addition, we achieve a priori estimates with explicit dependence on the time for the pressure of the acoustic wave and the displacement of the elastic wave by considering directly the time-domain variational problem and taking special test functions.

The paper is organized as follows. In section 2, we introduce the model equations and interface conditions for the acoustic-elastic interaction problem. The time-domain TBC is presented and some trace results are proved. Section 3 is devoted to the analysis of the reduced problem, where the well-posdeness and stability are addressed in both the frequency and time domains. We conclude the paper with some remarks in section 4

2. Problem formulation. In this section, we define some notation, introduce the model equations, and present an initial-boundary value problem for the acousticelastic scattering in an air/fluid-solid medium.

2.1. Problem Geometry. As shown in Figure 2.1 we consider an active source which is embedded in an elastic solid medium. It models an earthquake focus located in the crust which lies between the lithosphere and the rigid mantle of the Earth. Due to the excitation of the source, an elastic wave is generated in the solid and propagates 


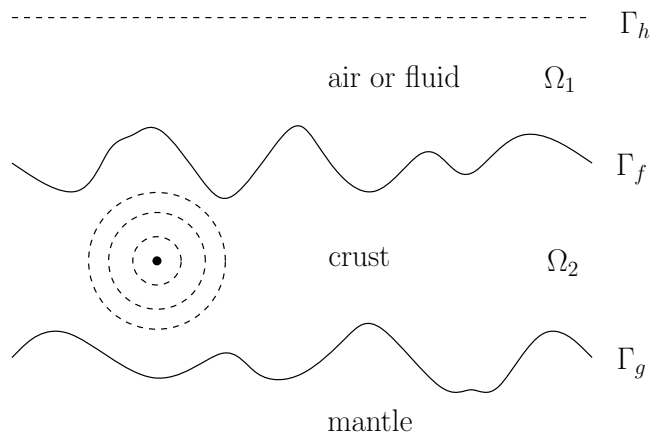

FIG. 2.1. Problem geometry of the acoustic-elastic interaction in an unbounded structure.

through to the medium of the air/fluid. Clearly, this process leads to the air/fluidsolid interaction problem with the scattering interface separating the domains where the acoustic and elastic waves travel.

Let $\boldsymbol{r}=(x, y)^{\top} \in \mathbb{R}^{2}$ and $\boldsymbol{x}=(x, y, z)^{\top} \in \mathbb{R}^{3}$. Denote by $\Gamma_{f}=\left\{\boldsymbol{x} \in \mathbb{R}^{3}: z=\right.$ $f(\boldsymbol{r})\}$ the surface separating the air/fluid and the solid, where $f$ is assumed to be a $W^{1, \infty}\left(\mathbb{R}^{2}\right)$ function. Let $\Gamma_{g}=\left\{\boldsymbol{x} \in \mathbb{R}^{3}: z=g(\boldsymbol{r})\right\}$ be the surface separating the crust and the mantle, where $g$ is a $L^{\infty}\left(\mathbb{R}^{2}\right)$ function satisfying $g(\boldsymbol{r})<f(\boldsymbol{r}), \boldsymbol{r} \in \mathbb{R}^{2}$. We assume that the open space $\Omega_{f}^{+}=\left\{\boldsymbol{x} \in \mathbb{R}^{3}: z>f(\boldsymbol{r})\right\}$ is filled with a homogeneous compressible air or a compressible inviscid fluid with the constant density $\rho_{1}$. The space $\Omega_{2}=\left\{\boldsymbol{x} \in \mathbb{R}^{3}: g(\boldsymbol{r}) \leq z \leq f(\boldsymbol{r})\right\}$ is assumed to be occupied by a homogeneous isotropic linear elastic solid which is characterized by the constant mass density $\rho_{2}$ and Lamé parameters $\mu, \lambda$. Define an artificial planar surface $\Gamma_{h}=\left\{\boldsymbol{x} \in \mathbb{R}^{3}, z=h\right\}$, where $h>\sup _{\boldsymbol{r} \in \mathbb{R}^{2}} f(\boldsymbol{r})$ is a constant. Let $\Omega_{1}=\left\{\boldsymbol{x} \in \mathbb{R}^{3}: f(\boldsymbol{r})<z<h\right\}$ and $\Omega=\Omega_{1} \cup \Omega_{2}$.

2.2. Acoustic wave equation. The acoustic wave field in air/fluid is governed by the conservation and the dynamics equations in the time-domain:

$$
\nabla p(\boldsymbol{x}, t)=-\rho_{1} \partial_{t} \boldsymbol{v}(\boldsymbol{x}, t), \quad c^{2} \rho_{1} \nabla \cdot \boldsymbol{v}(\boldsymbol{x}, t)=-\partial_{t} p(\boldsymbol{x}, t), \quad \boldsymbol{x} \in \Omega_{f}^{+}, t>0,
$$

where $p$ is the pressure, $\boldsymbol{v}$ is the velocity, and the constants $\rho_{1}>0$ and $c>0$ are the density and sound speed, respectively. Eliminating the velocity $v$ from (2.1), we obtain the acoustic wave equation for the pressure $p$ :

$$
\Delta p(\boldsymbol{x}, t)-\frac{1}{c^{2}} \partial_{t}^{2} p(\boldsymbol{x}, t)=0, \quad \boldsymbol{x} \in \Omega_{f}^{+}, t>0
$$

The equation is constrained by the homogeneous initial conditions:

$$
\left.p\right|_{t=0}=0,\left.\quad \partial_{t} p\right|_{t=0}=0, \quad \boldsymbol{x} \in \Omega_{f}^{+} .
$$

It follows from the conservation equation in 2.1) that $\nabla \times \boldsymbol{v}(\boldsymbol{x}, t)=0$, i.e., the acoustic air/fluid is irrotational. Thus there exists a scalar potential function $\varphi$ such that $\boldsymbol{v}(\boldsymbol{x}, t)=\nabla \varphi(\boldsymbol{x})$. It is easy to note from (2.1) that the corresponding dynamic component of the pressure is given by

$$
p(\boldsymbol{x}, t)=-\rho_{1} \partial_{t} \varphi(\boldsymbol{x}, t) .
$$


2.3. Elastic wave equation. For the solid, the elastic wave field in a homogeneous isotropic solid material satisfies the linear time-domain elasticity equation:

$$
\nabla \cdot \boldsymbol{\sigma}(\boldsymbol{u}(\boldsymbol{x}, t))-\rho_{2} \partial_{t}^{2} \boldsymbol{u}(\boldsymbol{x}, t)=\boldsymbol{j}(\boldsymbol{x}, t), \quad \boldsymbol{x} \in \Omega_{2}, t>0,
$$

where $\boldsymbol{u}=\left(u_{1}, u_{2}, u_{3}\right)^{\top}$ is the displacement vector, $\rho_{2}>0$ is the density of the elastic solid material, $\boldsymbol{j}$ is the source which models the earthquake focus and is assumed to have a compact support contained in $\Omega_{2}$, and the symmetric stress tensor $\boldsymbol{\sigma}(\boldsymbol{u})$ is given by the generalized Hook law:

$$
\boldsymbol{\sigma}(\boldsymbol{u})=2 \mu \mathcal{E}(\boldsymbol{u})+\lambda \operatorname{tr}(\mathcal{E}(\boldsymbol{u})) \mathrm{I}, \quad \mathcal{E}(\boldsymbol{u})=\frac{1}{2}\left(\nabla \boldsymbol{u}+(\nabla \boldsymbol{u})^{\top}\right) .
$$

Here $\mu, \lambda$ are the Lamé parameters satisfying $\mu>0, \lambda+\mu>0, \mathrm{I} \in \mathbb{R}^{3 \times 3}$ is the identity matrix $\operatorname{tr}(\mathcal{E}(\boldsymbol{u}))$ is the trace of the matrix $\mathcal{E}(\boldsymbol{u})$, and $\nabla \boldsymbol{u}$ is the displacement gradient tensor given by

$$
\nabla \boldsymbol{u}=\left[\begin{array}{lll}
\partial_{x} u_{1} & \partial_{y} u_{1} & \partial_{z} u_{1} \\
\partial_{x} u_{2} & \partial_{y} u_{2} & \partial_{z} u_{2} \\
\partial_{x} u_{3} & \partial_{y} u_{3} & \partial_{z} u_{3}
\end{array}\right]
$$

Substituting (2.3) into (2.2), we obtain the time-domain Navier equation for the displacement $\boldsymbol{u}$ :

$$
\mu \Delta \boldsymbol{u}(\boldsymbol{x}, t)+(\lambda+\mu) \nabla \nabla \cdot \boldsymbol{u}(\boldsymbol{x}, t)-\rho_{2} \partial_{t}^{2} \boldsymbol{u}(\boldsymbol{x}, t)=\boldsymbol{j}(\boldsymbol{x}, t), \quad \boldsymbol{x} \in \Omega_{2}, t>0 .
$$

By assuming that the mantle is rigid, we have

$$
\boldsymbol{u}=0 \quad \text { on } \Gamma_{g}, t>0 .
$$

The elastic wave equation (2.4) is constrained by the homogeneous initial conditions:

$$
\left.\boldsymbol{u}\right|_{t=0}=0,\left.\quad \partial_{t} \boldsymbol{u}\right|_{t=0}=0, \quad \boldsymbol{x} \in \Omega_{2}
$$

2.4. Interface conditions. To couple the acoustic wave equation in the air/fluid and the elastic wave equation in the solid, the kinematic interface condition is imposed to ensure the continuity of the normal component of the velocity on $\Gamma_{f}$ :

$$
\boldsymbol{n} \cdot \boldsymbol{v}(\boldsymbol{x}, t)=\boldsymbol{n} \cdot \partial_{t} \boldsymbol{u}(\boldsymbol{x}, t), \quad \boldsymbol{x} \in \Gamma_{f}, t>0,
$$

where $\boldsymbol{n}$ is the unit normal on $\Gamma_{f}$ pointing from $\Omega_{2}$ to $\Omega_{1}$. Noting $\boldsymbol{v}(\boldsymbol{x}, t)=\nabla \varphi(\boldsymbol{x}, t)$ and $p(\boldsymbol{x}, t)=-\rho_{1} \partial_{t} \varphi(\boldsymbol{x}, t)$, we have from (2.5) that

$$
\partial_{\boldsymbol{n}} p=\boldsymbol{n} \cdot \nabla p=-\rho_{1} \boldsymbol{n} \cdot \partial_{t}^{2} \boldsymbol{u} \quad \text { on } \Gamma_{f}, t>0 .
$$

In addition, the dynamic interface condition is required to ensure the continuity of traction:

$$
-p \boldsymbol{n}=\boldsymbol{\sigma}(\boldsymbol{u}) \cdot \boldsymbol{n}, \quad \boldsymbol{x} \in \Gamma_{f}, t>0,
$$

where $\boldsymbol{\sigma}(\boldsymbol{u}) \cdot \boldsymbol{n}$ is denoted as the multiplication of the stress tensor $\boldsymbol{\sigma}(\boldsymbol{u})$ with the normal vector $\boldsymbol{n}$. 
2.5. Laplace transform and some functional spaces. We first introduce some properties of the Laplace transform. For any $s=s_{1}+\mathrm{i} s_{2}$ with $s_{1}>0, s_{2} \in \mathbb{R}$, define $\breve{\boldsymbol{u}}(s)$ to be the Laplace transform of the function $\boldsymbol{u}(t)$, i.e.,

$$
\breve{\boldsymbol{u}}(s)=\mathscr{L}(\boldsymbol{u})(s)=\int_{0}^{\infty} e^{-s t} \boldsymbol{u}(t) \mathrm{d} t .
$$

It follows from the integration by parts that

$$
\int_{0}^{t} \boldsymbol{u}(\tau) \mathrm{d} \tau=\mathscr{L}^{-1}\left(s^{-1} \breve{\boldsymbol{u}}(s)\right)
$$

where $\mathscr{L}^{-1}$ is the inverse Laplace transform. It is easy to verify from the inverse Laplace transform that

$$
\boldsymbol{u}(t)=\mathscr{F}^{-1}\left(e^{s_{1} t} \mathscr{L}(\boldsymbol{u})\left(s_{1}+s_{2}\right)\right),
$$

where $\mathscr{F}^{-1}$ denotes the inverse Fourier transform with respect to $s_{2}$.

Recall the Plancherel or Parseval identity for the Laplace transform (cf. [, (2.46)]):

$$
\frac{1}{2 \pi} \int_{-\infty}^{\infty} \breve{\boldsymbol{u}}(s) \cdot \breve{\boldsymbol{v}}(s) \mathrm{d} s_{2}=\int_{0}^{\infty} e^{-2 s_{1} t} \boldsymbol{u}(t) \cdot \boldsymbol{v}(t) \mathrm{d} t, \quad \forall s_{1}>\lambda,
$$

where $\breve{\boldsymbol{u}}=\mathscr{L}(\boldsymbol{u}), \breve{\boldsymbol{v}}=\mathscr{L}(\boldsymbol{v})$ and $\lambda$ is the abscissa of convergence for the Laplace transform of $\boldsymbol{u}$ and $\boldsymbol{v}$.

The following lemma (cf. [34. Theorem 43.1]) is an analogue of Paley-WienerSchwarz theorem for the Fourier transform of the distributions with compact supports in the case of the Laplace transform.

Lemma 2.1. Let $\breve{\boldsymbol{h}}(s)$ be a holomorphic function in the half-plane $s_{1}>\sigma_{0}$ and be valued in the Banach space $\mathbb{E}$. The following two conditions are equivalent:

1. there is a distribution $\boldsymbol{h} \in \mathcal{D}_{+}^{\prime}(\mathbb{E})$ whose Laplace transform is equal to $\breve{\boldsymbol{h}}(s)$;

2. there is a real $\sigma_{1}$ with $\sigma_{0} \leq \sigma_{1}<\infty$ and an integer $m \geq 0$ such that for all complex numbers $s$ with $\operatorname{Re} s=s_{1}>\sigma_{1}$, we have $\|\breve{h}(s)\|_{\mathbb{E}} \lesssim(1+|s|)^{m}$,

where $\mathcal{D}_{+}^{\prime}(\mathbb{E})$ is the space of distributions on the real line which vanish identically in the open negative half line.

Next we introduce some Sobolev spaces. For any $u(\cdot, h) \in L^{2}\left(\Gamma_{h}\right)$ which is identified as $L^{2}\left(\mathbb{R}^{2}\right)$, we denote by $\hat{u}(\boldsymbol{\xi}, h)$ the Fourier transform of $u(\boldsymbol{r}, h)$ :

$$
\hat{u}(\boldsymbol{\xi}, h)=\frac{1}{2 \pi} \int_{\mathbb{R}^{2}} u(\boldsymbol{r}, h) e^{-\mathrm{i} \boldsymbol{r} \cdot \boldsymbol{\xi}} \mathrm{d} \boldsymbol{r},
$$

where $\boldsymbol{\xi}=\left(\xi_{1}, \xi_{2}\right)^{\top} \in \mathbb{R}^{2}$. For any $\alpha \in \mathbb{R}$, define the functional space

$$
H^{\alpha}\left(\Gamma_{h}\right)=\left\{u(\boldsymbol{r}, h) \in L^{2}\left(\mathbb{R}^{2}\right): \int_{\mathbb{R}^{2}}\left(1+|\boldsymbol{\xi}|^{2}\right)^{\alpha}|\hat{u}(\boldsymbol{\xi}, h)|^{2} \mathrm{~d} \boldsymbol{\xi}<\infty\right\},
$$

which is a Sobolev space under the norm

$$
\|u\|_{H^{\alpha}\left(\Gamma_{h}\right)}=\left[\int_{\mathbb{R}^{2}}\left(1+|\boldsymbol{\xi}|^{2}\right)^{\alpha}|\hat{u}(\boldsymbol{\xi}, h)|^{2} \mathrm{~d} \boldsymbol{\xi}\right]^{1 / 2} .
$$

It is clear to note that the dual space associated with $H^{\alpha}\left(\Gamma_{h}\right)$ is the space $H^{-\alpha}\left(\Gamma_{h}\right)$ with respect to the scalar product in $L^{2}\left(\mathbb{R}^{2}\right)$ defined by

$$
\langle u, v\rangle_{\Gamma_{h}}=\int_{\Gamma_{h}} u(\boldsymbol{r}, h) \bar{v}(\boldsymbol{r}, h) \mathrm{d} \boldsymbol{r}=\int_{\mathbb{R}^{2}} \hat{u}(\boldsymbol{\xi}, h) \overline{\hat{v}}(\boldsymbol{\xi}, h) \mathrm{d} \boldsymbol{\xi} .
$$


Denote by $H^{1 / 2}\left(\Gamma_{f}\right)$ the Sobolev trace space, the subspace of $L^{2}\left(\Gamma_{f}\right)$ such that

$$
\int_{\Gamma_{f}}|u(\boldsymbol{r}, f(\boldsymbol{r}))|^{2} \mathrm{~d} \boldsymbol{r}+\int_{\Gamma_{f}} \int_{\Gamma_{f}} \frac{\left|u\left(\boldsymbol{r}_{1}, f\left(\boldsymbol{r}_{1}\right)\right)-u\left(\boldsymbol{r}_{2}, f\left(\boldsymbol{r}_{2}\right)\right)\right|^{2}}{\left|\boldsymbol{r}_{1}-\boldsymbol{r}_{2}\right|^{3}} \mathrm{~d} \boldsymbol{r}_{1} \mathrm{~d} \boldsymbol{r}_{2}<\infty .
$$

$H^{1 / 2}\left(\Gamma_{f}\right)$ is equipped with the norm

$\|u\|_{H^{1 / 2}\left(\Gamma_{f}\right)}=\left(\int_{\Gamma_{f}}|u(\boldsymbol{r}, f(\boldsymbol{r}))|^{2} \mathrm{~d} \boldsymbol{r}+\int_{\Gamma_{f}} \int_{\Gamma_{f}} \frac{\left|u\left(\boldsymbol{r}_{1}, f\left(\boldsymbol{r}_{1}\right)\right)-u\left(\boldsymbol{r}_{2}, f\left(\boldsymbol{r}_{2}\right)\right)\right|^{2}}{\left|\boldsymbol{r}_{1}-\boldsymbol{r}_{2}\right|^{3}} \mathrm{~d} \boldsymbol{r}_{1} \mathrm{~d} \boldsymbol{r}_{2}\right)^{1 / 2}$.

Denote by $H^{\nu}(\Omega)=\left\{D^{\alpha} u \in L^{2}(\Omega)\right.$ for all $\left.|\alpha| \leq \nu\right\}$ the standard Sobolev space of square integrable functions with the order of derivatives up to $\nu$. Let $H_{\Gamma_{g}}^{1}(\Omega)=\{u \in$ $H^{1}(\Omega): u=0$ on $\left.\Gamma_{g}\right\}$. Let $H_{\Gamma_{g}}^{1}(\Omega)^{3}$ and $H^{1 / 2}\left(\Gamma_{f}\right)^{3}$ be the Cartesian product spaces equipped with the corresponding 2-norms of $H_{\Gamma_{g}}^{1}(\Omega)$ and $H^{1 / 2}\left(\Gamma_{f}\right)$, respectively. For any $\boldsymbol{u}=\left(u_{1}, u_{2}, u_{3}\right)^{\top} \in H_{\Gamma_{g}}^{1}\left(\Omega_{2}\right)^{3}$, define the Frobenius norm:

$$
\|\nabla \boldsymbol{u}\|_{F\left(\Omega_{2}\right)}=\left(\sum_{j=1}^{3} \int_{\Omega_{2}}\left|\nabla u_{j}\right|^{2} \mathrm{~d} \boldsymbol{x}\right)^{1 / 2}
$$

It is easy to verify that

$$
\|\nabla \boldsymbol{u}\|_{F\left(\Omega_{2}\right)}^{2}+\|\nabla \cdot \boldsymbol{u}\|_{L^{2}\left(\Omega_{2}\right)}^{2} \lesssim\|\boldsymbol{u}\|_{H^{1}\left(\Omega_{2}\right)^{3}}^{2} .
$$

Hereafter, the expression $a \lesssim b$ or $a \gtrsim c$ stands for $a \leq C b$ or $a \geq C b$, where $C$ is a positive constant and its specific value is not required but should be always clear from the context.

2.6. Transparent boundary condition. In this subsection, we will introduce an exact time-domain TBC to formulate the acoustic-elastic wave interaction problem into the following coupled initial-boundary value problem:

$$
\begin{cases}\Delta p-\frac{1}{c^{2}} \partial_{t}^{2} p=0 & \text { in } \Omega_{1}, t>0 \\ \mu \Delta \boldsymbol{u}+(\lambda+\mu) \nabla \nabla \cdot \boldsymbol{u}-\rho_{2} \partial_{t}^{2} \boldsymbol{u}=\boldsymbol{j} & \text { in } \Omega_{2}, t>0, \\ \left.p\right|_{t=0}=\left.\partial_{t} p\right|_{t=0}=0,\left.\quad \boldsymbol{u}\right|_{t=0}=\left.\partial_{t} \boldsymbol{u}\right|_{t=0}=0 & \text { in } \Omega, \\ \partial_{\boldsymbol{n}} p=-\rho_{1} \boldsymbol{n} \cdot \partial_{t}^{2} \boldsymbol{u}, \quad-p \boldsymbol{n}=\boldsymbol{\sigma}(\boldsymbol{u}) \cdot \boldsymbol{n} & \text { on } \Gamma_{f}, t>0, \\ \partial_{\boldsymbol{\nu}} p=\mathscr{T}_{p}, & \text { on } \Gamma_{h}, t>0, \\ \boldsymbol{u}=0 & \text { on } \Gamma_{g}, t>0,\end{cases}
$$

where $\boldsymbol{\nu}=(0,0,1)^{\top}$ is the unit normal vector on $\Gamma_{h}$ pointing from $\Omega_{1}$ to $\Omega_{h}^{+}=\{\boldsymbol{x} \in$ $\left.\mathbb{R}^{2}: z>h\right\}$, and $\mathscr{T}$ is the time-domain TBC operator on $\Gamma_{h}$. In what follows, we shall derive the formulation of the operator $\mathscr{T}$ and show some of its properties.

Let $\breve{p}(\boldsymbol{x}, s)=\mathscr{L}(p)$ and $\breve{\boldsymbol{u}}(\boldsymbol{x}, s)=\mathscr{L}(\boldsymbol{u})$ be the Laplace transform of $p(\boldsymbol{x}, t)$ and $\boldsymbol{u}(\boldsymbol{x}, t)$ with respect to $t$, respectively. Recall that

$$
\begin{aligned}
& \mathscr{L}\left(\partial_{t} p\right)=s \breve{p}(\cdot, s)-p(\cdot, 0), \quad \mathscr{L}\left(\partial_{t}^{2} p\right)=s^{2} \breve{p}(\cdot, s)-s p(\cdot, 0)-\partial_{t} p(\cdot, 0), \\
& \mathscr{L}\left(\partial_{t} \boldsymbol{u}\right)=s \breve{\boldsymbol{u}}(\cdot, s)-\boldsymbol{u}(\cdot, 0), \quad \mathscr{L}\left(\partial_{t}^{2} \boldsymbol{u}\right)=s^{2} \breve{\boldsymbol{u}}(\cdot, s)-s \boldsymbol{u}(\cdot, 0)-\partial_{t} \boldsymbol{u}(\cdot, 0)
\end{aligned}
$$


Taking the Laplace transform of (2.8) and using the initial conditions, we obtain the acoustic-elastic wave interaction problem in the $s$-domain:

$$
\begin{cases}\Delta \breve{p}-\frac{s^{2}}{c^{2}} \breve{p}=0 & \text { in } \Omega_{1}, \\ \mu \Delta \breve{\boldsymbol{u}}+(\lambda+\mu) \nabla \nabla \cdot \breve{\boldsymbol{u}}-\rho_{2} s^{2} \breve{\boldsymbol{u}}=\breve{\boldsymbol{j}} & \text { in } \Omega_{2}, \\ \partial_{\boldsymbol{n}} \breve{p}=-\rho_{1} s^{2} \boldsymbol{n} \cdot \breve{\boldsymbol{u}}, \quad-\breve{p} \boldsymbol{n}=\boldsymbol{\sigma}(\breve{\boldsymbol{u}}) \cdot \boldsymbol{n} & \text { on } \Gamma_{f}, \\ \partial_{\boldsymbol{\nu}} \breve{p}=\mathscr{B} \breve{p}, & \text { on } \Gamma_{h}, \\ \breve{\boldsymbol{u}}=0 & \text { on } \Gamma_{g},\end{cases}
$$

where $\breve{j}=\mathscr{L}(\boldsymbol{j}), \mathscr{B}$ is the Dirichlet-to-Neumann (DtN) operator on $\Gamma_{h}$ in $s$-domain and satisfies $\mathscr{T}=\mathscr{L}^{-1} \circ \mathscr{B} \circ \mathscr{L}$.

In order to deduce the TBC, we consider the Helmholtz equation with a complex wavenumber:

$$
\Delta \breve{p}-\frac{s^{2}}{c^{2}} \breve{p}=0 \quad \text { in } \Omega_{h}^{+} .
$$

Taking the Fourier transform of (2.9) with respect to $\boldsymbol{r}$ yields

$$
\begin{cases}\frac{\mathrm{d}^{2} \hat{p}(\boldsymbol{\xi}, z)}{\mathrm{d} z^{2}}-\left(\frac{s^{2}}{c^{2}}+|\boldsymbol{\xi}|^{2}\right) \hat{p}(\boldsymbol{\xi}, z)=0, & z>h, \\ \hat{\grave{p}}(\boldsymbol{\xi}, z)=\hat{\grave{p}}(\boldsymbol{\xi}, h), & z=h .\end{cases}
$$

Solving (2.10) and using the bounded outgoing wave condition, we get

$$
\hat{\grave{p}}(\boldsymbol{\xi}, z)=\hat{\grave{p}}(\boldsymbol{\xi}, h) e^{-\beta(\boldsymbol{\xi})(z-h)}, \quad z>h,
$$

where

$$
\beta^{2}(\boldsymbol{\xi})=\frac{s^{2}}{c^{2}}+|\boldsymbol{\xi}|^{2} \quad \text { with } \operatorname{Re} \beta(\boldsymbol{\xi})>0 .
$$

Thus we obtain the solution of (2.9):

$$
\breve{p}(\boldsymbol{r}, z)=\int_{\mathbb{R}^{2}} \hat{\grave{p}}(\boldsymbol{\xi}, h) e^{-\beta(\boldsymbol{\xi})(z-h)} e^{\mathrm{i} \boldsymbol{\xi} \cdot \boldsymbol{r}} \mathrm{d} \boldsymbol{\xi} .
$$

Taking the normal derivative of (2.12) on $\Gamma_{h}$ and evaluating it at $z=h$, we have

$$
\partial_{\boldsymbol{\nu}} \breve{p}(\boldsymbol{r}, h)=\int_{\mathbb{R}^{2}}-\beta(\boldsymbol{\xi}) \hat{\tilde{p}}(\boldsymbol{\xi}, h) e^{\mathrm{i} \boldsymbol{\xi} \cdot \boldsymbol{r}} \mathrm{d} \boldsymbol{\xi}
$$

For any function $u(\boldsymbol{r}, h)$ defined on $\Gamma_{h}$, we defined the DtN operator

$$
(\mathscr{B} u)(\boldsymbol{r}, h)=\int_{\mathbb{R}^{2}}-\beta(\boldsymbol{\xi}) \hat{u}(\boldsymbol{\xi}, h) e^{\mathrm{i} \boldsymbol{\xi} \cdot \boldsymbol{r}} \mathrm{d} \boldsymbol{\xi} .
$$

Let $z_{1}, z_{2}$ be two constants satisfying $z_{2}<z_{1}$. Define $\Gamma_{j}=\left\{\boldsymbol{x} \in \mathbb{R}^{2}: z=z_{j}\right\}$ and $R=\left\{\boldsymbol{x} \in \mathbb{R}^{3}: \boldsymbol{r} \in \mathbb{R}^{2}, z_{2}<z<z_{1}\right\}$. The following several trace results are useful in subsequent analysis.

Lemma 2.2. Let $\gamma_{0}=\left(1+\left(z_{1}-z_{2}\right)^{-1}\right)^{1 / 2}$. We have the estimate

$$
\|u\|_{H^{1 / 2}\left(\Gamma_{j}\right)} \leq \gamma_{0}\|u\|_{H^{1}(R)}, \quad \forall u \in H^{1}(R) .
$$


Proof. First we have

$$
\begin{aligned}
\left(z_{1}-z_{2}\right)\left|\zeta\left(z_{j}\right)\right|^{2} & =\int_{z_{2}}^{z_{1}}|\zeta(z)|^{2} \mathrm{~d} z+\int_{z_{2}}^{z_{1}} \int_{z}^{z_{j}} \frac{\mathrm{d}}{\mathrm{d} \tau}|\zeta(\tau)|^{2} \mathrm{~d} \tau \mathrm{d} z \\
& \leq \int_{z_{2}}^{z_{1}}|\zeta(z)|^{2} \mathrm{~d} z+\left(z_{1}-z_{2}\right) \int_{z_{2}}^{z_{1}} 2|\zeta(z)|\left|\zeta^{\prime}(z)\right| \mathrm{d} z
\end{aligned}
$$

which implies by the Cauchy-Schwarz inequality that

$$
\left(1+|\boldsymbol{\xi}|^{2}\right)^{1 / 2}\left|\zeta\left(z_{j}\right)\right|^{2} \leq \gamma_{0}^{2}\left(1+|\boldsymbol{\xi}|^{2}\right) \int_{z_{2}}^{z_{1}}|\zeta(z)|^{2} \mathrm{~d} z+\int_{z_{2}}^{z_{1}}\left|\zeta^{\prime}(z)\right|^{2} \mathrm{~d} z .
$$

Given $u$ in $H^{1}(R)$, a simple calculation yields that

$$
\|u\|_{H^{1 / 2}\left(\Gamma_{j}\right)}^{2}=\int_{\mathbb{R}^{2}}\left(1+|\boldsymbol{\xi}|^{2}\right)^{1 / 2}\left|\hat{u}\left(\boldsymbol{\xi}, z_{j}\right)\right|^{2} \mathrm{~d} \boldsymbol{\xi}
$$

and

$$
\|u\|_{H^{1}(R)}^{2}=\int_{z_{2}}^{z_{1}} \int_{\mathbb{R}^{2}}\left[\left(1+|\boldsymbol{\xi}|^{2}\right)|\hat{u}(\boldsymbol{\xi}, z)|^{2}+\left|\hat{u}^{\prime}(\boldsymbol{\xi}, z)\right|^{2}\right] \mathrm{d} \boldsymbol{\xi} \mathrm{d} z,
$$

where $\hat{u}^{\prime}(\boldsymbol{\xi}, z)=\partial_{z} \hat{u}(\boldsymbol{\xi}, z)$.

Using (2.14), we obtain

$$
\begin{aligned}
\left(1+|\boldsymbol{\xi}|^{2}\right)^{1 / 2}\left|\hat{u}\left(\boldsymbol{\xi}, z_{j}\right)\right|^{2} & \leq \gamma_{0}^{2}\left(1+|\boldsymbol{\xi}|^{2}\right) \int_{z_{2}}^{z_{1}}|\hat{u}(\boldsymbol{\xi}, z)|^{2} \mathrm{~d} z+\int_{z_{2}}^{z_{1}}\left|\hat{u}^{\prime}(\boldsymbol{\xi}, z)\right|^{2} \mathrm{~d} z \\
& \leq \gamma_{0}^{2} \int_{z_{2}}^{z_{1}}\left[\left(1+|\boldsymbol{\xi}|^{2}\right)|\hat{u}(\boldsymbol{\xi}, z)|^{2}+\left|\hat{u}^{\prime}(\boldsymbol{\xi}, z)\right|^{2}\right] \mathrm{d} z,
\end{aligned}
$$

which completes the proof after combining (2.15) and (2.16).

LEMma 2.3. There exists a positive constant $C$ such that

$$
\|u\|_{H^{1 / 2}\left(\Gamma_{f}\right)} \leq C\|u\|_{H^{1}\left(\Omega_{1}\right)}, \quad \forall u \in H^{1}\left(\Omega_{1}\right) .
$$

Proof. Consider the change of variables:

$$
\tilde{x}=x, \quad \tilde{y}=y, \quad \tilde{z}=h\left(\frac{z-f}{h-f}\right),
$$

which maps the domain $\Omega_{1}$ into the rectangular slab $D_{1}:=\left\{\tilde{\boldsymbol{x}}=(\tilde{x}, \tilde{y}, \tilde{z}) \in \mathbb{R}^{3}\right.$ : $0<\tilde{z}<h\}$. In particular, the surface $\Gamma_{f}$ is transformed to the planar surface $\Gamma_{0}:=\left\{\tilde{\boldsymbol{x}} \in \mathbb{R}^{3}: \tilde{z}=0\right\}$. Let $J$ be the Jacobian matrix of the transformation. A simple calculation yields that

$$
|J|=\left|\frac{\partial(\tilde{x}, \tilde{y}, \tilde{z})}{\partial(x, y, z)}\right|=\left|\begin{array}{ccc}
1 & 0 & 0 \\
0 & 1 & 0 \\
\frac{h(z-h) \partial_{x} f}{(h-f)^{2}} & \frac{h(z-h) \partial_{y} f}{(h-f)^{2}} & \frac{h}{h-f}
\end{array}\right|=\frac{h}{h-f} \neq 0,
$$

which shows that the transformation is invertible. Denote by $J^{-1}$ the inverse of the Jacobian matrix. It follows from Lemma 2.2 that we have

$$
\|u\|_{H^{1 / 2}\left(\Gamma_{0}\right)} \lesssim\|u\|_{H^{1}\left(D_{1}\right)} .
$$


Using the usual Sobolev norm in $\Omega_{1}$ and the change of variables, we get

$$
\begin{aligned}
\|u\|_{H^{1}\left(\Omega_{1}\right)}^{2}= & \int_{\Omega_{1}}\left(|u(\boldsymbol{x})|^{2}+|\nabla u(\boldsymbol{x})|^{2}\right) \mathrm{d} \boldsymbol{x} \\
= & \int_{D_{1}}\left[|u|^{2}+\left|\partial_{\tilde{x}} u-\partial_{\tilde{x}} f\left(\frac{h-\tilde{z}}{h-f}\right) \partial_{\tilde{z}} u\right|^{2}\right. \\
& \left.\quad+\left|\partial_{\tilde{y}} u-\partial_{\tilde{y}} f\left(\frac{h-\tilde{z}}{h-f}\right) \partial_{\tilde{z} u}\right|^{2}+\left|\left(\frac{h}{h-f}\right) \partial_{\tilde{z}} u\right|^{2}\right] J^{-1} \mathrm{~d} \tilde{\boldsymbol{x}} \\
\lesssim & \int_{D_{1}}\left(|u(\tilde{\boldsymbol{x}})|^{2}+|\nabla u(\tilde{\boldsymbol{x}})|^{2}\right) \mathrm{d} \tilde{\boldsymbol{x}}=\|u\|_{H^{1}\left(D_{1}\right)}^{2},
\end{aligned}
$$

where we have used the assumption that $f \in W^{1, \infty}\left(\mathbb{R}^{2}\right)$. On the other hand, we have

$$
\begin{aligned}
\|u\|_{H^{1}\left(D_{1}\right)}^{2}= & \int_{D_{1}}\left(|u(\tilde{\boldsymbol{x}})|^{2}+|\nabla u(\tilde{\boldsymbol{x}})|^{2}\right) \mathrm{d} \tilde{\boldsymbol{x}} \\
= & \int_{\Omega_{1}}\left[|u|^{2}+\left|\partial_{x} u+\partial_{x} f\left(\frac{h-z}{h-f}\right) \partial_{z} u\right|^{2}\right. \\
& \left.\quad+\left|\partial_{y} u+\partial_{y} f\left(\frac{h-z}{h-f}\right) \partial_{z} u\right|^{2}+\left(\partial_{z} u \frac{h-f}{h}\right)^{2}\right] J \mathrm{~d} \boldsymbol{x} \\
\lesssim & \int_{\Omega_{1}}\left(|u(\boldsymbol{x})|^{2}+|\nabla u(\boldsymbol{x})|^{2}\right) \mathrm{d} \boldsymbol{x}=\|u\|_{H^{1}\left(\Omega_{1}\right)}^{2} .
\end{aligned}
$$

Combining (2.18) and (2.19), we get that the norm $\|u\|_{H^{1}\left(\Omega_{1}\right)}^{2}$ is equivalent to the norm $\|u\|_{H^{1}\left(D_{1}\right)}^{2}$.

Next, we prove the equivalence of the norm $\|u\|_{H^{1 / 2}\left(\Gamma_{0}\right)}$ and the norm $\|u\|_{H^{1 / 2}\left(\Gamma_{f}\right)}$. First we have

$$
\|u\|_{H^{1 / 2}\left(\Gamma_{0}\right)}^{2}=\int_{\Gamma_{0}}|u(\tilde{\boldsymbol{r}}, 0)|^{2} \mathrm{~d} \tilde{\boldsymbol{r}}+\int_{\Gamma_{0}} \int_{\Gamma_{0}} \frac{\left|u\left(\tilde{\boldsymbol{r}}_{1}, 0\right)-u\left(\tilde{\boldsymbol{r}}_{2}, 0\right)\right|^{2}}{\left|\tilde{\boldsymbol{r}}_{1}-\tilde{\boldsymbol{r}}_{2}\right|^{3}} \mathrm{~d} \tilde{\boldsymbol{r}}_{1} \mathrm{~d} \tilde{\boldsymbol{r}}_{2} .
$$

It follows from the change of variables that we have

$$
\begin{aligned}
\|u\|_{H^{1 / 2}\left(\Gamma_{f}\right)}^{2}= & \int_{\Gamma_{f}}|u(\boldsymbol{r}, f(\boldsymbol{r}))|^{2} \mathrm{~d} \boldsymbol{r}+\int_{\Gamma_{f}} \int_{\Gamma_{f}} \frac{\left|u\left(\boldsymbol{r}_{1}, f\left(\boldsymbol{r}_{1}\right)\right)-u\left(\boldsymbol{r}_{2}, f\left(\boldsymbol{r}_{2}\right)\right)\right|^{2}}{\left|\boldsymbol{r}_{1}-\boldsymbol{r}_{2}\right|^{3}} \mathrm{~d} \boldsymbol{r}_{1} \mathrm{~d} \boldsymbol{r}_{2} \\
= & \int_{\Gamma_{0}}|u(\tilde{\boldsymbol{r}}, 0)|^{2}\left(1+\left|\nabla_{\tilde{\boldsymbol{r}}} f\right|^{2}\right)^{1 / 2} \mathrm{~d} \tilde{\boldsymbol{r}}+\int_{\Gamma_{0}} \int_{\Gamma_{0}} \frac{\left|u\left(\tilde{\boldsymbol{r}}_{1}, 0\right)-u\left(\tilde{\boldsymbol{r}}_{2}, 0\right)\right|^{2}}{\left|\tilde{\boldsymbol{r}}_{1}-\tilde{\boldsymbol{r}}_{2}\right|^{3}} \\
& \times\left(1+\left|\nabla_{\tilde{\boldsymbol{r}}_{1}} f\right|^{2}\right)^{1 / 2}\left(1+\left|\nabla_{\tilde{\boldsymbol{r}}_{2}} f\right|^{2}\right)^{1 / 2} \mathrm{~d} \tilde{\boldsymbol{r}}_{1} \mathrm{~d} \tilde{\boldsymbol{r}}_{2} .
\end{aligned}
$$

Hence we obtain

$$
\|u\|_{H^{1 / 2}\left(\Gamma_{0}\right)} \leq\|u\|_{H^{1 / 2}\left(\Gamma_{f}\right)} \lesssim\|u\|_{H^{1 / 2}\left(\Gamma_{0}\right)} .
$$

The proof is completed by using (2.17) and the equivalence of the norms.

Lemma 2.4. There exists a positive constant $C$ such that

$$
\|\boldsymbol{u}\|_{H^{1 / 2}\left(\Gamma_{f}\right)^{3}} \leq C\|\boldsymbol{u}\|_{H^{1}\left(\Omega_{2}\right)^{3}}, \quad \forall \boldsymbol{u} \in H_{\Gamma_{g}}^{1}\left(\Omega_{2}\right)^{3} .
$$


Proof. Denote $\tilde{\Omega}_{2}=\left\{\boldsymbol{x} \in \mathbb{R}^{3}: \boldsymbol{r} \in \mathbb{R}^{2}, \inf _{\boldsymbol{r} \in \mathbb{R}^{2}} g(\boldsymbol{r})<z<f(\boldsymbol{r})\right\}$ which contains the domain $\Omega_{2}$. For any $\boldsymbol{u} \in H_{\Gamma_{g}}^{1}\left(\Omega_{2}\right)^{3}$, we consider the zero extension to $\tilde{\Omega}_{2}$ :

$$
\tilde{\boldsymbol{u}}(\boldsymbol{x})= \begin{cases}\boldsymbol{u}(\boldsymbol{x}), & \boldsymbol{x} \in \Omega_{2}, \\ 0, & \boldsymbol{x} \in \tilde{\Omega}_{2} \backslash \bar{\Omega}_{2} .\end{cases}
$$

It is clear to note that

$$
\|\boldsymbol{u}\|_{H^{1 / 2}\left(\Gamma_{f}\right)^{3}}=\|\tilde{\boldsymbol{u}}\|_{H^{1 / 2}\left(\Gamma_{f}\right)^{3}}, \quad\|\boldsymbol{u}\|_{H^{1}\left(\Omega_{2}\right)^{3}}=\|\tilde{\boldsymbol{u}}\|_{H^{1}\left(\tilde{\Omega}_{2}\right)^{3}} .
$$

It follows from Lemmas 2.2 and 2.3 that there exists a positive constant $C$ such that

$$
\|\tilde{\boldsymbol{u}}\|_{H^{1 / 2}\left(\Gamma_{f}\right)^{3}} \leq C\|\tilde{\boldsymbol{u}}\|_{H^{1}\left(\tilde{\Omega}_{2}\right)^{3}} .
$$

Combining (2.20) and (2.21) completes the proof. $\mathrm{Q}$

Lemma 2.5. The DtN operator $\mathscr{B}: H^{1 / 2}\left(\Gamma_{h}\right) \rightarrow H^{-1 / 2}\left(\Gamma_{h}\right)$ is continuous, i.e.,

$$
\|\mathscr{B} u\|_{H^{-1 / 2}\left(\Gamma_{h}\right)} \lesssim\|u\|_{H^{1 / 2}\left(\Gamma_{h}\right)}, \quad \forall u \in H^{1 / 2}\left(\Gamma_{h}\right) .
$$

Proof. For any $u \in H^{1 / 2}\left(\Gamma_{h}\right)$, it follows from (2.13) and (2.11) that

$$
\begin{aligned}
\|\mathscr{B} u\|_{H^{-1 / 2}\left(\Gamma_{h}\right)}^{2} & =\int_{\mathbb{R}^{2}}\left(1+|\boldsymbol{\xi}|^{2}\right)^{-1 / 2}|-\beta(\boldsymbol{\xi}) \hat{u}(\boldsymbol{\xi}, h)|^{2} \mathrm{~d} \boldsymbol{\xi} \\
& =\int_{\mathbb{R}^{2}}\left(1+|\boldsymbol{\xi}|^{2}\right)^{1 / 2}\left(1+|\boldsymbol{\xi}|^{2}\right)^{-1}|\beta(\boldsymbol{\xi})|^{2}|\hat{u}(\boldsymbol{\xi}, h)|^{2} \mathrm{~d} \boldsymbol{\xi} \lesssim\|u\|_{H^{1 / 2}\left(\Gamma_{h}\right)}^{2},
\end{aligned}
$$

where we have used

$$
|\beta(\boldsymbol{\xi})|^{2}=\left.\left.\left|\frac{s^{2}}{c^{2}}+\right| \boldsymbol{\xi}\right|^{2}\left|\leq \frac{|s|^{2}}{c^{2}}+\right| \boldsymbol{\xi}\right|^{2} \lesssim 1+|\boldsymbol{\xi}|^{2},
$$

which completes the proof. $\square$

LEMMA 2.6. We have

$$
-\operatorname{Re}\left\langle s^{-1} \mathscr{B} u, u\right\rangle_{\Gamma_{h}} \geq 0, \quad \forall u \in H^{1 / 2}\left(\Gamma_{h}\right) .
$$

Proof. A simple calculation yields that

$$
-\left\langle s^{-1} \mathscr{B} u, u\right\rangle_{\Gamma_{h}}=\int_{\mathbb{R}^{2}} s^{-1} \beta(\boldsymbol{\xi})|\hat{u}(\boldsymbol{\xi}, h)|^{2} \mathrm{~d} \boldsymbol{\xi}=\int_{\mathbb{R}^{2}} \frac{\bar{s} \beta(\boldsymbol{\xi})}{|s|^{2}}|\hat{u}(\boldsymbol{\xi}, h)|^{2} \mathrm{~d} \boldsymbol{\xi}
$$

Let $\beta(\boldsymbol{\xi})=a+\mathrm{i} b, s=s_{1}+\mathrm{i} s_{2}$ with $a>0, s_{1}>0$. Taking the real part of the above equation gives

$$
-\operatorname{Re}\left\langle s^{-1} \mathscr{B} u, u\right\rangle_{\Gamma_{h}}=\int_{\mathbb{R}^{2}} \frac{\left(s_{1} a+s_{2} b\right)}{|s|^{2}}|\hat{u}(\boldsymbol{\xi}, h)|^{2} \mathrm{~d} \boldsymbol{\xi} .
$$

Recalling $\beta^{2}(\boldsymbol{\xi})=\frac{s^{2}}{c^{2}}+|\boldsymbol{\xi}|^{2}$, we have

$$
a^{2}-b^{2}=\frac{s_{1}^{2}-s_{2}^{2}}{c^{2}}+|\boldsymbol{\xi}|^{2}, \quad a b=\frac{s_{1} s_{2}}{c^{2}} .
$$


Substituting (2.23) into (2.22) yields

$$
-\operatorname{Re}\left\langle s^{-1} \mathscr{B} u, u\right\rangle_{\Gamma_{h}}=\int_{\mathbb{R}^{2}} \frac{1}{|s|^{2}}\left(a s_{1}+\frac{s_{1}}{a} \frac{s_{2}^{2}}{c^{2}}\right)|\hat{u}(\boldsymbol{\xi}, h)|^{2} \mathrm{~d} \boldsymbol{\xi} \geq 0,
$$

which completes the proof.

For any function $u(\boldsymbol{r}, h)$ defined on $\Gamma_{h}$, using the DtN operator (2.13), we can obtain the following TBC in the $s$-domain:

$$
\partial_{\nu} \breve{p}=\mathscr{B} \breve{p} \quad \text { on } \Gamma_{h} .
$$

Taking the inverse Laplace transform of (2.24) yields the TBC in the time-domain:

$$
\partial_{\nu} p=\mathscr{T} p \quad \text { on } \Gamma_{h} .
$$

3. The reduced problem. In this section, we present the main results of this paper, which include the well-posedness and stability of the scattering problem and related a priori estimates.

3.1. Well-posedness in the $s$-domain. Consider the reduced problem in the $s$-domain:

$$
\begin{cases}\Delta \breve{p}-\frac{s^{2}}{c^{2}} \breve{p}=0 & \text { in } \Omega_{1}, \\ \mu \Delta \breve{\boldsymbol{u}}+(\lambda+\mu) \nabla \nabla \cdot \breve{\boldsymbol{u}}-\rho_{2} s^{2} \breve{\boldsymbol{u}}=\breve{\boldsymbol{j}} & \text { in } \Omega_{2}, \\ \partial_{\boldsymbol{n}} \breve{p}=-\rho_{1} s^{2} \boldsymbol{n} \cdot \breve{\boldsymbol{u}}, \quad-\breve{p} \boldsymbol{n}=\boldsymbol{\sigma}(\breve{\boldsymbol{u}}) \cdot \boldsymbol{n} & \text { on } \Gamma_{f}, \\ \partial_{\boldsymbol{\nu}} \breve{p}=\mathscr{B} \breve{p}, & \text { on } \Gamma_{h}, \\ \breve{\boldsymbol{u}}=0 & \text { on } \Gamma_{g} .\end{cases}
$$

Multiplying (3.1a) and (3.1b) by the complex conjugate of a test function $q \in H^{1}\left(\Omega_{1}\right)$ and a test function $\boldsymbol{v} \in H_{\Gamma_{g}}^{1}\left(\Omega_{2}\right)^{3}$, respectively, using the integration by parts and boundary conditions, which include the TBC condition (3.1d), the kinematic and dynamic interface conditions (3.1c), and the rigid boundary condition (3.1e), we arrive at the variational problem: To find $(\breve{p}, \breve{\boldsymbol{u}}) \in H^{1}\left(\Omega_{1}\right) \times H_{\Gamma_{g}}^{1}\left(\Omega_{2}\right)^{3}$ such that

$$
\int_{\Omega_{1}}\left(\frac{1}{s} \nabla \breve{p} \cdot \nabla \bar{q}+\frac{s}{c^{2}} \breve{p} \bar{q}\right) \mathrm{d} \boldsymbol{x}-\left\langle s^{-1} \mathscr{B} \breve{p}, q\right\rangle_{\Gamma_{h}}-\rho_{1} s \int_{\Gamma_{f}}(\boldsymbol{n} \cdot \breve{\boldsymbol{u}}) \bar{q} \mathrm{~d} \gamma=0
$$

and

$$
\begin{aligned}
& \int_{\Omega_{2}} \frac{1}{s}\left((\mu(\nabla \breve{\boldsymbol{u}}: \nabla \overline{\boldsymbol{v}})+(\lambda+\mu)(\nabla \cdot \breve{\boldsymbol{u}})(\nabla \cdot \overline{\boldsymbol{v}}))+\rho_{2} s \breve{\boldsymbol{u}} \cdot \overline{\boldsymbol{v}}\right) \mathrm{d} \boldsymbol{x} \\
& \quad+\frac{1}{s} \int_{\Gamma_{f}} \breve{p}(\boldsymbol{n} \cdot \overline{\boldsymbol{v}}) \mathrm{d} \gamma=-\int_{\Omega_{2}} \frac{1}{s} \breve{\boldsymbol{j}} \cdot \overline{\boldsymbol{v}} \mathrm{d} \boldsymbol{x}, \quad \forall(q, \boldsymbol{v}) \in H^{1}\left(\Omega_{1}\right) \times H_{\Gamma_{g}}^{1}\left(\Omega_{2}\right),
\end{aligned}
$$

where $A: B=\operatorname{tr}\left(A B^{\top}\right)$ is the Frobenius inner product of square matrices $A$ and $B$.

We multiply (3.3) by $\rho_{1}|s|^{2}$ and add the obtained result to (3.2) to obtain an equivalent variational problem: To find $(\breve{p}, \breve{\boldsymbol{u}}) \in H^{1}\left(\Omega_{1}\right) \times H_{\Gamma_{g}}^{1}\left(\Omega_{2}\right)^{3}$ such that

$$
a(\breve{p}, \breve{\boldsymbol{u}} ; q, \boldsymbol{v})=-\int_{\Omega_{2}} \rho_{1} \bar{s} \breve{\boldsymbol{j}} \cdot \overline{\boldsymbol{v}} \mathrm{d} \boldsymbol{x}, \quad \forall(q, \boldsymbol{v}) \in H^{1}\left(\Omega_{1}\right) \times H_{\Gamma_{g}}^{1}\left(\Omega_{2}\right)^{3},
$$


where the sesquilinear form

$$
\begin{aligned}
a(\breve{p}, \breve{\boldsymbol{u}} ; q, \boldsymbol{v})= & \int_{\Omega_{1}}\left(\frac{1}{s} \nabla \breve{p} \cdot \nabla \bar{q}+\frac{s}{c^{2}} \breve{p} \bar{q}\right) \mathrm{d} \boldsymbol{x}+\int_{\Omega_{2}}\left(\rho_{1} \bar{s}(\mu(\nabla \breve{\boldsymbol{u}}: \nabla \overline{\boldsymbol{v}})\right. \\
& \left.+(\lambda+\mu)(\nabla \cdot \breve{\boldsymbol{u}})(\nabla \cdot \overline{\boldsymbol{v}}))+\rho_{1} \rho_{2} s|s|^{2} \breve{\boldsymbol{u}} \cdot \overline{\boldsymbol{v}}\right) \mathrm{d} \boldsymbol{x}-\left\langle s^{-1} \mathscr{B} \breve{p}, q\right\rangle_{\Gamma_{h}} \\
& +\rho_{1} \int_{\Gamma_{f}}(\bar{s} \breve{p}(\boldsymbol{n} \cdot \overline{\boldsymbol{v}})-s \bar{q}(\boldsymbol{n} \cdot \breve{\boldsymbol{u}})) \mathrm{d} \gamma .
\end{aligned}
$$

THEOREM 3.1. The variational problem (3.4) has a unique weak solution $(\breve{p}, \breve{\boldsymbol{u}}) \in$ $H^{1}\left(\Omega_{1}\right) \times H_{\Gamma_{g}}^{1}\left(\Omega_{2}\right)^{3}$, which satisfies

$$
\begin{aligned}
\|\nabla \breve{p}\|_{L^{2}\left(\Omega_{1}\right)^{3}}+\|s \breve{p}\|_{L^{2}\left(\Omega_{1}\right)} & \lesssim\|\breve{j}\|_{L^{2}\left(\Omega_{2}\right)^{3}}, \\
\|\nabla \breve{\boldsymbol{u}}\|_{F\left(\Omega_{2}\right)}+\|\nabla \cdot \breve{\boldsymbol{u}}\|_{L^{2}\left(\Omega_{2}\right)}+\|s \breve{\boldsymbol{u}}\|_{L^{2}\left(\Omega_{2}\right)^{3}} & \lesssim \frac{1}{|s|}\|\breve{\boldsymbol{j}}\|_{L^{2}\left(\Omega_{2}\right)^{3}}
\end{aligned}
$$

Proof. We have from the Cauchy-Schwarz inequality and Lemmas 2.2 2.5 that

$$
\begin{gathered}
|a(\breve{p}, \breve{\boldsymbol{u}} ; q, \boldsymbol{v})| \leq \frac{1}{|s|}\|\nabla \breve{p}\|_{L^{2}\left(\Omega_{1}\right)^{3}}\|\nabla q\|_{L^{2}\left(\Omega_{1}\right)^{3}}+\frac{|s|}{c^{2}}\|\breve{p}\|_{L^{2}\left(\Omega_{1}\right)}\|q\|_{L^{2}\left(\Omega_{1}\right)} \\
+\rho_{1}|s|\left(\mu\|\nabla \breve{\boldsymbol{u}}\|_{F\left(\Omega_{2}\right)}\|\nabla \boldsymbol{v}\|_{F\left(\Omega_{2}\right)}+(\lambda+\mu)\|\nabla \cdot \breve{\boldsymbol{u}}\|_{L^{2}(\Omega)}\|\nabla \cdot \boldsymbol{v}\|_{L^{2}(\Omega)}\right) \\
+\rho_{1} \rho_{2}|s|^{3}\|\breve{\boldsymbol{u}}\|_{L^{2}\left(\Omega_{2}\right)^{3}}\|\boldsymbol{v}\|_{L^{2}\left(\Omega_{2}\right)^{3}}+\frac{1}{|s|}\|\mathscr{B} \breve{p}\|_{H^{-1 / 2}\left(\Gamma_{h}\right)}\|q\|_{H^{1 / 2}\left(\Gamma_{h}\right)} \\
\quad+\rho_{1}|s|\left(\|p\|_{L^{2}\left(\Gamma_{f}\right)}\|\boldsymbol{n} \cdot \boldsymbol{v}\|_{L^{2}\left(\Gamma_{f}\right)}+\|q\|_{L^{2}\left(\Gamma_{f}\right)}\|\boldsymbol{n} \cdot \breve{\boldsymbol{u}}\|_{L^{2}\left(\Gamma_{f}\right)}\right) \\
\lesssim\|\breve{p}\|_{H^{1}\left(\Omega_{1}\right)}\|q\|_{H^{1}\left(\Omega_{1}\right)}+\|\breve{\boldsymbol{u}}\|_{H^{1}\left(\Omega_{2}\right)^{3}}\|\boldsymbol{v}\|_{H^{1}\left(\Omega_{2}\right)^{3}}+\|\breve{p}\|_{H^{1 / 2}\left(\Gamma_{h}\right)}\|q\|_{H^{1 / 2}\left(\Gamma_{h}\right)} \\
\quad+\|\breve{p}\|_{H^{1 / 2}\left(\Gamma_{f}\right)}\|\boldsymbol{v}\|_{H^{1 / 2}\left(\Gamma_{f}\right)^{3}}+\|q\|_{H^{1 / 2}\left(\Gamma_{f}\right)}\|\breve{\boldsymbol{u}}\|_{H^{1 / 2}\left(\Gamma_{f}\right)^{3}} \\
\lesssim\|\breve{p}\|_{H^{1}\left(\Omega_{1}\right)}\|q\|_{H^{1}\left(\Omega_{1}\right)}+\|\breve{\boldsymbol{u}}\|_{H^{1}\left(\Omega_{2}\right)^{3}}\|\boldsymbol{v}\|_{H^{1}\left(\Omega_{2}\right)^{3}}+\|\breve{p}\|_{H^{1}\left(\Omega_{1}\right)}\|q\|_{H^{1}\left(\Omega_{1}\right)} \\
+\|\breve{p}\|_{H^{1}\left(\Omega_{1}\right)}\|\boldsymbol{v}\|_{H^{1}\left(\Omega_{2}\right)^{3}}+\|q\|_{H^{1}\left(\Omega_{1}\right)}\|\breve{\boldsymbol{u}}\|_{H^{1}\left(\Omega_{2}\right)^{3}},
\end{gathered}
$$

which shows that the sesquilinear form is bounded.

Letting $(q, \boldsymbol{v})=(\breve{p}, \breve{\boldsymbol{u}})$ in (3.5) yields

$a(\breve{p}, \breve{\boldsymbol{u}} ; \breve{p}, \breve{\boldsymbol{u}})=\int_{\Omega_{1}}\left(\frac{1}{s}|\nabla \breve{p}|^{2}+\frac{s}{c^{2}}|\breve{p}|^{2}\right) \mathrm{d} \boldsymbol{x}+\int_{\Omega_{2}}\left(\rho_{1} \bar{s}\left(\mu(\nabla \breve{\boldsymbol{u}}: \nabla \overline{\boldsymbol{u}})+(\lambda+\mu)|\nabla \cdot \breve{\boldsymbol{u}}|^{2}\right)\right.$

$$
\left.+\rho_{1} \rho_{2} s|s|^{2}|\breve{\boldsymbol{u}}|^{2}\right) \mathrm{d} \boldsymbol{x}-\left\langle s^{-1} \mathscr{B} \breve{p}, \breve{p}\right\rangle_{\Gamma_{h}}+\rho_{1} \int_{\Gamma_{f}}(\bar{s} \breve{p}(\boldsymbol{n} \cdot \overline{\boldsymbol{u}})-s \bar{p}(\boldsymbol{n} \cdot \breve{\boldsymbol{u}})) \mathrm{d} \gamma .
$$

Taking the real part of (3.8) and using Lemma 2.6] we obtain

$$
\begin{aligned}
\operatorname{Re}(a(\breve{p}, \breve{\boldsymbol{u}} ; \breve{p}, \breve{\boldsymbol{u}}))= & \int_{\Omega_{1}}\left(\frac{s_{1}}{|s|^{2}}|\nabla \breve{p}|^{2}+\frac{s_{1}}{c^{2}}|\breve{p}|^{2}\right) \mathrm{d} \boldsymbol{x}+\rho_{1} s_{1}\left(\|\nabla \breve{\boldsymbol{u}}\|_{F\left(\Omega_{2}\right)}^{2}\right. \\
& \left.\quad+(\lambda+\mu)\|\nabla \cdot \breve{\boldsymbol{u}}\|_{L^{2}\left(\Omega_{2}\right)}^{2}\right)+\rho_{1} \rho_{2} s_{1}|s|^{2}|\breve{\boldsymbol{u}}|_{L^{2}\left(\Omega_{2}\right)^{3}}^{2}-\operatorname{Re}\left\langle s^{-1} \mathscr{B} \breve{p}, \breve{p}\right\rangle_{\Gamma_{h}} \\
\gtrsim & \frac{s_{1}}{|s|^{2}}\left(\|\nabla \breve{p}\|_{L^{2}\left(\Omega_{1}\right)^{3}}^{2}+\|s \breve{p}\|_{L^{2}\left(\Omega_{1}\right)}^{2}\right) \\
& \quad+s_{1}\left(\|\nabla \breve{\boldsymbol{u}}\|_{F\left(\Omega_{2}\right)}^{2}+\|\nabla \cdot \breve{\boldsymbol{u}}\|_{L^{2}\left(\Omega_{2}\right)}^{2}+\|s \breve{\boldsymbol{u}}\|_{L^{2}\left(\Omega_{2}\right)^{3}}^{2}\right) .
\end{aligned}
$$

It follows from the Lax-Milgram lemma that the variational problem (3.4) has a unique weak solution $(\breve{p}, \breve{u}) \in H^{1}\left(\Omega_{1}\right) \times H_{\Gamma_{g}}^{1}\left(\Omega_{2}\right)^{3}$. 
Moreover, we have from (3.4) that

$$
|a(\breve{p}, \breve{\boldsymbol{u}} ; \breve{p}, \breve{\boldsymbol{u}})| \lesssim \frac{s_{1}}{|s|}\|\breve{\boldsymbol{j}}\|_{L^{2}\left(\Omega_{2}\right)^{3}}\|s \breve{\boldsymbol{u}}\|_{L^{2}\left(\Omega_{2}\right)^{3}}
$$

Combing (3.9) and (3.10) leads to

$$
\begin{aligned}
\|\nabla \breve{\boldsymbol{u}}\|_{F\left(\Omega_{2}\right)}^{2} & +\|\nabla \cdot \breve{\boldsymbol{u}}\|_{L^{2}\left(\Omega_{2}\right)}^{2}+\|s \breve{\boldsymbol{u}}\|_{L^{2}\left(\Omega_{2}\right)^{3}}^{2} \\
& \lesssim \frac{1}{s_{1}}|a(\breve{p}, \breve{\boldsymbol{u}} ; \breve{p}, \breve{\boldsymbol{u}})| \lesssim \frac{1}{|s|}\|\breve{\boldsymbol{j}}\|_{L^{2}\left(\Omega_{2}\right)^{3}}\|s \breve{\boldsymbol{u}}\|_{L^{2}\left(\Omega_{2}\right)^{3}}
\end{aligned}
$$

and

$$
\begin{aligned}
\frac{1}{|s|^{2}}\left(\|\nabla \breve{p}\|_{L^{2}\left(\Omega_{1}\right)^{3}}^{2}\right. & \left.+\|s \breve{p}\|_{L^{2}\left(\Omega_{1}\right)}^{2}\right)+|s \breve{\boldsymbol{u}}|_{L^{2}\left(\Omega_{2}\right)^{3}}^{2} \\
& \lesssim \frac{1}{s_{1}}|a(\breve{p}, \breve{\boldsymbol{u}} ; \breve{p}, \breve{\boldsymbol{u}})| \lesssim \frac{1}{|s|}\|\breve{\boldsymbol{j}}\|_{L^{2}\left(\Omega_{2}\right)^{3}}\|s \breve{\boldsymbol{u}}\|_{L^{2}\left(\Omega_{2}\right)^{3}}
\end{aligned}
$$

Using the Cauchy-Schwarz inequality, we obtain

$$
\|\nabla \breve{\boldsymbol{u}}\|_{F\left(\Omega_{2}\right)}+\|\nabla \cdot \breve{\boldsymbol{u}}\|_{L^{2}\left(\Omega_{2}\right)}+\|s \breve{\boldsymbol{u}}\|_{L^{2}\left(\Omega_{2}\right)^{3}} \lesssim \frac{1}{|s|}\|\breve{\boldsymbol{j}}\|_{L^{2}\left(\Omega_{2}\right)^{3}}
$$

and

$$
\begin{aligned}
\frac{1}{|s|}\left(\|\nabla \breve{p}\|_{L^{2}\left(\Omega_{1}\right)^{3}}+\|s \breve{p}\|_{L^{2}\left(\Omega_{1}\right)}\right) & \lesssim \frac{1}{|s|}\left(\|\nabla \breve{p}\|_{L^{2}\left(\Omega_{1}\right)^{3}}+\|s \breve{p}\|_{L^{2}\left(\Omega_{1}\right)}\right)+\|s \breve{\boldsymbol{u}}\|_{L^{2}\left(\Omega_{2}\right)^{3}} \\
& \lesssim \frac{1}{|s|}\|\breve{j}\|_{L^{2}\left(\Omega_{2}\right)^{3}}
\end{aligned}
$$

which completes the proof.

3.2. Well-posedness in the time-domain. We now consider the reduced problem in the time-domain:

$$
\begin{cases}\Delta p-\frac{1}{c^{2}} \partial_{t}^{2} p=0 & \text { in } \Omega_{1}, t>0 \\ \mu \Delta \boldsymbol{u}+(\lambda+\mu) \nabla \nabla \cdot \boldsymbol{u}-\rho_{2} \partial_{t}^{2} \boldsymbol{u}=\boldsymbol{j} & \text { in } \Omega_{2}, t>0 \\ \left.p\right|_{t=0}=\left.\partial_{t} p\right|_{t=0}=0,\left.\quad \boldsymbol{u}\right|_{t=0}=\left.\partial_{t} \boldsymbol{u}\right|_{t=0}=0 & \text { in } \Omega, \\ \partial_{\boldsymbol{n}} p=-\rho_{1} \boldsymbol{n} \cdot \partial_{t}^{2} \boldsymbol{u}, \quad-p \boldsymbol{n}=\boldsymbol{\sigma}(\boldsymbol{u}) \cdot \boldsymbol{n} & \text { on } \Gamma_{f}, t>0 \\ \partial_{\boldsymbol{\nu}} p=\mathscr{T}_{p} & \text { on } \Gamma_{h}, t>0 \\ \boldsymbol{u}=0 & \text { on } \Gamma_{g}, t>0 .\end{cases}
$$

To show the well-posedness of the reduced problem (3.11), we make the following assumption for the source term $\boldsymbol{j}$ :

$$
\boldsymbol{j} \in H^{1}\left(0, T ; L^{2}\left(\Omega_{2}\right)^{3}\right),\left.\quad \boldsymbol{j}\right|_{t=0}=0 .
$$

THEOREM 3.2. The initial-boundary value problem (3.11) has a unique solution $(p, \boldsymbol{u})$ which satisfies

$$
\begin{aligned}
& p(\boldsymbol{x}, t) \in L^{2}\left(0, T ; H^{1}\left(\Omega_{1}\right)\right) \cap H^{1}\left(0, T ; L^{2}\left(\Omega_{1}\right)\right), \\
& \boldsymbol{u}(\boldsymbol{x}, t) \in L^{2}\left(0, T ; H_{\Gamma_{g}}^{1}\left(\Omega_{2}\right)^{3}\right) \cap H^{1}\left(0, T ; L^{2}\left(\Omega_{2}\right)^{3}\right)
\end{aligned}
$$


and the stability estimates

$$
\begin{aligned}
& \max _{t \in[0, T]}\left(\left\|\partial_{t} p\right\|_{L^{2}\left(\Omega_{1}\right)}+\|\nabla p\|_{L^{2}\left(\Omega_{1}\right)^{3}}\right) \lesssim\left\|\partial_{t} \boldsymbol{j}\right\|_{L^{1}\left(0, T ; L^{2}\left(\Omega_{2}\right)^{3}\right)}, \\
& \max _{t \in[0, T]}\left(\left\|\partial_{t} \boldsymbol{u}\right\|_{L^{2}\left(\Omega_{2}\right)^{3}}+\|\nabla \cdot \boldsymbol{u}\|_{L^{2}\left(\Omega_{2}\right)}+\|\nabla \boldsymbol{u}\|_{F\left(\Omega_{2}\right)}\right) \lesssim\left\|\partial_{t} \boldsymbol{j}\right\|_{L^{1}\left(0, T ; L^{2}\left(\Omega_{2}\right)^{3}\right)} .
\end{aligned}
$$

Proof. For the air/fluid pressure $p$, we have

$$
\begin{aligned}
\int_{0}^{T} & \left(\|\nabla p\|_{L^{2}\left(\Omega_{1}\right)^{3}}^{2}+\left\|\partial_{t} p\right\|_{L^{2}\left(\Omega_{1}\right)}^{2}\right) \mathrm{d} t \\
& \leq \int_{0}^{T} e^{-2 s_{1}(t-T)}\left(\|\nabla p\|_{L^{2}\left(\Omega_{1}\right)^{3}}^{2}+\left\|\partial_{t} p\right\|_{L^{2}\left(\Omega_{1}\right)}^{2}\right) \mathrm{d} t \\
& =e^{2 s_{1} T} \int_{0}^{T} e^{-2 s_{1} t}\left(\|\nabla p\|_{L^{2}\left(\Omega_{1}\right)^{3}}^{2}+\left\|\partial_{t} p\right\|_{L^{2}\left(\Omega_{1}\right)}^{2}\right) \mathrm{d} t \\
& \lesssim \int_{0}^{\infty} e^{-2 s_{1} t}\left(\|\nabla p\|_{L^{2}\left(\Omega_{1}\right)^{2}}^{3}+\left\|\partial_{t} p\right\|_{L^{2}\left(\Omega_{1}\right)}^{2}\right) \mathrm{d} t .
\end{aligned}
$$

Similarly, we have for the elastic displacement $\boldsymbol{u}$ that

$$
\begin{aligned}
\int_{0}^{T} & \left(\left\|\partial_{t} \boldsymbol{u}\right\|_{L^{2}\left(\Omega_{2}\right)^{3}}^{2}+\|\nabla \boldsymbol{u}\|_{F\left(\Omega_{2}\right)}^{2}+\|\nabla \cdot \boldsymbol{u}\|_{L^{2}\left(\Omega_{2}\right)}^{2}\right) \mathrm{d} t \\
& \leq \int_{0}^{T} e^{-2 s_{1}(t-T)}\left(\left\|\partial_{t} \boldsymbol{u}\right\|_{L^{2}\left(\Omega_{2}\right)^{3}}^{2}+\|\nabla \boldsymbol{u}\|_{F\left(\Omega_{2}\right)}^{2}+\|\nabla \cdot \boldsymbol{u}\|_{L^{2}\left(\Omega_{2}\right)}^{2}\right) \mathrm{d} t \\
& =e^{2 s_{1} T} \int_{0}^{T} e^{-2 s_{1} t}\left(\left\|\partial_{t} \boldsymbol{u}\right\|_{L^{2}\left(\Omega_{2}\right)^{3}}^{2}+\|\nabla \boldsymbol{e}\|_{F\left(\Omega_{2}\right)}^{2}+\|\nabla \cdot \boldsymbol{u}\|_{L^{2}\left(\Omega_{2}\right)}^{2}\right) \mathrm{d} t \\
& \lesssim \int_{0}^{\infty} e^{-2 s_{1} t}\left(\left\|\partial_{t} \boldsymbol{u}\right\|_{L^{2}\left(\Omega_{2}\right)^{3}}^{2}+\|\nabla \boldsymbol{u}\|_{F\left(\Omega_{2}\right)}^{2}+\|\nabla \cdot \boldsymbol{u}\|_{L^{2}\left(\Omega_{2}\right)}^{2}\right) \mathrm{d} t .
\end{aligned}
$$

Hence it suffices to estimate the integrals

$$
\int_{0}^{\infty} e^{-2 s_{1} t}\left(\|\nabla p\|_{L^{2}\left(\Omega_{1}\right)^{3}}^{2}+\left\|\partial_{t} p\right\|_{L^{2}\left(\Omega_{1}\right)}^{2}\right) \mathrm{d} t
$$

and

$$
\int_{0}^{\infty} e^{-2 s_{1} t}\left(\left\|\partial_{t} \boldsymbol{u}\right\|_{L^{2}\left(\Omega_{2}\right)^{3}}^{2}+\|\nabla \boldsymbol{u}\|_{F\left(\Omega_{2}\right)}^{2}+\|\nabla \cdot \boldsymbol{u}\|_{L^{2}\left(\Omega_{2}\right)}^{2}\right) \mathrm{d} t .
$$

Taking the Laplace transform of (3.11), we obtain the reduced acoustic-elastic interaction problem in the $s$-domain (3.1). It follows from Theorem 3.1 that $\breve{p}$ and $\breve{\boldsymbol{u}}$ satisfy the stability estimates (3.6) and (3.7), respectively. It follows from [34, Lemma 44.1] that $\breve{p}$ and $\breve{\boldsymbol{u}}$ are holomorphic functions of $s$ on the half plane $s_{1}>\bar{\gamma}>0$, where $\bar{\gamma}$ is any positive constant. Hence we have from Lemma 2.1 that the inverse Laplace transform of $\breve{p}$ and $\breve{\boldsymbol{u}}$ exist and are supported in $[0, \infty]$.

Using the Parseval identity (2.6), the assumptions (3.12), and the stability esti- 
mate (3.6), we have

$$
\begin{aligned}
\int_{0}^{\infty} e^{-2 s_{1} t} & \left(\|\nabla p\|_{L^{2}\left(\Omega_{1}\right)^{3}}^{2}+\left\|\partial_{t} p\right\|_{L^{2}\left(\Omega_{1}\right)}^{2}\right) \mathrm{d} t=\frac{1}{2 \pi} \int_{-\infty}^{\infty}\left(\|\nabla \breve{p}\|_{L^{2}\left(\Omega_{1}\right)^{3}}^{2}+\|s \breve{p}\|_{L^{2}\left(\Omega_{1}\right)}^{2}\right) \mathrm{d} s_{2} \\
& \lesssim s_{1}^{-2} \int_{-\infty}^{\infty}\||s| \breve{j}\|_{L^{2}\left(\Omega_{2}\right)^{3}}^{2} \mathrm{~d} s_{2}=s_{1}^{-2} \int_{-\infty}^{\infty}\left\|\mathscr{L}\left(\partial_{t} \boldsymbol{j}\right)\right\|_{L^{2}\left(\Omega_{2}\right)^{3}}^{2} \mathrm{~d} s_{2} \\
& \lesssim s_{1}^{-2} \int_{0}^{\infty} e^{-2 s_{1} t}\left\|\partial_{t} \boldsymbol{j}\right\|_{L^{2}\left(\Omega_{2}\right)^{3}}^{2} \mathrm{~d} t
\end{aligned}
$$

which shows that

$$
p(\boldsymbol{x}, t) \in L^{2}\left(0, T ; H^{1}\left(\Omega_{1}\right)\right) \cap H^{1}\left(0, T ; L^{2}\left(\Omega_{1}\right)\right) .
$$

Since $\breve{\boldsymbol{u}}=\mathscr{L}(\boldsymbol{u})=\mathscr{F}\left(e^{-s_{1} t} \boldsymbol{u}\right)$, where $\mathscr{F}$ is the Fourier transform in $s_{2}$, we have from the Parseval identity (2.6) and the stability estimate (3.7) that

$$
\begin{aligned}
\int_{0}^{\infty} & e^{-2 s_{1} t}\left(\left\|\partial_{t} \boldsymbol{u}\right\|_{L^{2}\left(\Omega_{2}\right)^{3}}^{2}+\|\nabla \boldsymbol{u}\|_{F\left(\Omega_{2}\right)}^{2}+\|\nabla \cdot \boldsymbol{u}\|_{L^{2}\left(\Omega_{2}\right)}^{2}\right) \mathrm{d} t \\
& =\frac{1}{2 \pi} \int_{-\infty}^{\infty}\left(\|s \breve{\boldsymbol{u}}\|_{L^{2}\left(\Omega_{2}\right)^{3}}^{2}+\|\nabla \breve{\boldsymbol{u}}\|_{F\left(\Omega_{2}\right)}^{2}+\|\nabla \cdot \breve{\boldsymbol{u}}\|_{L^{2}\left(\Omega_{2}\right)}^{2}\right) \mathrm{d} s_{2} \\
& \lesssim s_{1}^{-2} \int_{-\infty}^{\infty}\|\breve{\boldsymbol{j}}\|_{L^{2}\left(\Omega_{2}\right)^{3}}^{2} \mathrm{~d} s_{2}=s_{1}^{-2} \int_{-\infty}^{\infty}\|\mathscr{L}(\boldsymbol{j})\|_{L^{2}\left(\Omega_{2}\right)^{3}}^{2} \mathrm{~d} s_{2} \\
& \lesssim s_{1}^{-2} \int_{0}^{\infty} e^{-2 s_{1} t}\|\boldsymbol{j}\|_{L^{2}\left(\Omega_{2}\right)^{3}}^{2} \mathrm{~d} t .
\end{aligned}
$$

It follows from (2.7) that

$$
\boldsymbol{u}(\boldsymbol{x}, t) \in L^{2}\left(0, T ; H_{\Gamma_{g}}^{1}\left(\Omega_{2}\right)^{3}\right) \cap H^{1}\left(0, T ; L^{2}\left(\Omega_{2}\right)^{3}\right) .
$$

Next we show the stability estimates. Let $\tilde{p}$ be the extension of $p$ with respect to $t$ in $\mathbb{R}$ such that $\breve{p}=0$ outside the interval $[0, t]$. By the Parseval identity (2.6) and Lemma 2.6. we get

$$
\begin{aligned}
& \operatorname{Re} \int_{0}^{t} e^{-2 s_{1} t}\left\langle\mathscr{T} p, \partial_{t} \bar{p}\right\rangle_{\Gamma_{h}} \mathrm{~d} t=\operatorname{Re} \int_{0}^{t} e^{-2 s_{1} t} \int_{\Gamma_{h}}(\mathscr{T} p) \partial_{t} \bar{p} \mathrm{~d} \boldsymbol{r} \mathrm{d} t \\
= & \operatorname{Re} \int_{\Gamma_{h}} \int_{0}^{\infty} e^{-2 s_{1} t}(\mathscr{T} \tilde{p}) \partial_{t} \overline{\tilde{p}} \mathrm{~d} t \mathrm{~d} \boldsymbol{r}=\frac{1}{2 \pi} \int_{-\infty}^{\infty} \operatorname{Re}\langle\mathscr{B} \breve{p}, s \breve{p}\rangle_{\Gamma_{h}} \mathrm{~d} s_{2} \\
= & \frac{1}{2 \pi} \int_{-\infty}^{\infty}|s|^{2} \operatorname{Re}\left\langle s^{-1} \mathscr{B} \breve{p}, \breve{p}\right\rangle_{\Gamma_{h}} \mathrm{~d} s_{2} \leq 0,
\end{aligned}
$$

which yields after taking $s_{1} \rightarrow 0$ that

$$
\operatorname{Re} \int_{0}^{t} \int_{\Gamma_{h}}(\mathscr{T} p) \partial_{t} \bar{p} \mathrm{~d} \boldsymbol{r} d t \leq 0 .
$$

Taking the partial derivative of (3.11b- $-3.11 \mathrm{~d}$ ) and (3.11f) with respect to $t$, we get

$$
\begin{cases}\mu \Delta\left(\partial_{t} \boldsymbol{u}\right)+(\lambda+\mu) \nabla \nabla \cdot\left(\partial_{t} \boldsymbol{u}\right)-\rho_{2} \partial_{t}^{2}\left(\partial_{t} \boldsymbol{u}\right)=\partial_{t} \boldsymbol{j} & \text { in } \Omega_{2}, t>0, \\ \left.\partial_{t} \boldsymbol{u}\right|_{t=0}=0 & \text { in } \Omega_{2}, \\ \left.\partial_{t}^{2} \boldsymbol{u}\right|_{t=0}=\left.\rho_{2}^{-1}(\mu \Delta \boldsymbol{u}+(\lambda+\mu) \nabla \nabla \cdot \boldsymbol{u}-\boldsymbol{j})\right|_{t=0}=0 & \text { in } \Omega_{2}, \\ -\partial_{t} p \boldsymbol{n}=\partial_{t}(\boldsymbol{\sigma}(\boldsymbol{u})) \cdot \boldsymbol{n}=\boldsymbol{\sigma}\left(\partial_{t} \boldsymbol{u}\right) \cdot \boldsymbol{n} & \text { on } \Gamma_{f}, t>0, \\ \partial_{t} \boldsymbol{u}=0 & \text { on } \Gamma_{g}, t>0 .\end{cases}
$$


For any $0<t<T$, consider the energy function

$$
\mathscr{E}(t)=e_{1}(t)+e_{2}(t)
$$

where

$$
e_{1}(t)=\left\|\frac{1}{c} \partial_{t} p\right\|_{L^{2}\left(\Omega_{1}\right)}^{2}+\|\nabla p\|_{L^{2}\left(\Omega_{1}\right)^{3}}^{2}
$$

and

$$
\begin{aligned}
e_{2}(t)=\left\|\left(\rho_{1} \rho_{2}\right)^{1 / 2} \partial_{t}^{2} \boldsymbol{u}\right\|_{L^{2}\left(\Omega_{2}\right)^{3}}^{2} & +\left\|\left(\rho_{1}(\lambda+\mu)\right)^{1 / 2} \nabla \cdot\left(\partial_{t} \boldsymbol{u}\right)\right\|_{L^{2}\left(\Omega_{2}\right)}^{2} \\
& +\left\|\left(\rho_{1} \mu\right)^{1 / 2} \nabla\left(\partial_{t} \boldsymbol{u}\right)\right\|_{F\left(\Omega_{2}\right)}^{2} .
\end{aligned}
$$

It is easy to note that

$$
\mathscr{E}(t)-\mathscr{E}(0)=\int_{0}^{t} \mathscr{E}^{\prime}(\tau) \mathrm{d} \tau=\int_{0}^{t}\left(e_{1}^{\prime}(\tau)+e_{2}^{\prime}(\tau)\right) \mathrm{d} \tau .
$$

It follows from (3.11a), (3.11c)-(3.11e) and the integration by parts that

$$
\begin{aligned}
\int_{0}^{t} e_{1}^{\prime}(\tau) \mathrm{d} \tau= & 2 \operatorname{Re} \int_{0}^{t} \int_{\Omega_{1}}\left(\frac{1}{c^{2}} \partial_{t}^{2} p \partial_{t} \bar{p}+\partial_{t}(\nabla p) \cdot \nabla \bar{p}\right) \mathrm{d} \boldsymbol{x} \mathrm{d} \tau \\
= & 2 \operatorname{Re} \int_{0}^{t} \int_{\Omega_{1}}\left(\Delta p \partial_{t} \bar{p}+\partial_{t}(\nabla p) \cdot \nabla \bar{p}\right) \mathrm{d} \boldsymbol{x} \mathrm{d} \tau \\
= & \int_{0}^{t} \int_{\Omega_{1}} 2 \operatorname{Re}\left(-\nabla p \cdot \partial_{t}(\nabla \bar{p})+\partial_{t}(\nabla p) \cdot \nabla \bar{p}\right) \mathrm{d} \boldsymbol{x} \mathrm{d} \tau \\
& \quad+2 \operatorname{Re} \int_{0}^{t} \int_{\Gamma_{h}}(\mathscr{T} p) \partial_{t} \bar{p} \mathrm{~d} \boldsymbol{d} \mathrm{d} \tau-2 \operatorname{Re} \int_{0}^{t} \int_{\Gamma_{f}} \partial_{\boldsymbol{n}} p \partial_{t} \bar{p} \mathrm{~d} \gamma \mathrm{d} \tau \\
= & 2 \operatorname{Re} \int_{0}^{t} \int_{\Gamma_{h}}(\mathscr{T} p) \partial_{t} \bar{p} \mathrm{~d} \boldsymbol{d} \tau+2 \operatorname{Re} \int_{0}^{t} \int_{\Gamma_{f}} \rho_{1} \boldsymbol{n} \cdot \partial_{t}^{2} \boldsymbol{u} \partial_{t} \bar{p} \mathrm{~d} \gamma \mathrm{d} \tau .
\end{aligned}
$$

Similarly, we have from (3.16) and the integration by parts that

$$
\begin{aligned}
\int_{0}^{t} e_{2}^{\prime}(\tau) \mathrm{d} \tau= & \rho_{1} 2 \operatorname{Re} \int_{0}^{t} \int_{\Omega_{2}}\left(\rho_{2} \partial_{t}\left(\partial_{t}^{2} \boldsymbol{u}\right) \cdot \partial_{t}^{2} \overline{\boldsymbol{u}}+(\lambda+\mu) \nabla \cdot\left(\partial_{t}^{2} \boldsymbol{u}\right) \nabla \cdot\left(\partial_{t} \overline{\boldsymbol{u}}\right)\right. \\
+ & \left.\mu \nabla\left(\partial_{t}^{2} \boldsymbol{u}\right): \nabla\left(\partial_{t} \overline{\boldsymbol{u}}\right)\right) \mathrm{d} \boldsymbol{x} \mathrm{d} \tau \\
= & \rho_{1} 2 \operatorname{Re} \int_{0}^{t} \int_{\Omega_{2}}\left(\left(\mu \Delta\left(\partial_{t} \boldsymbol{u}\right)+(\lambda+\mu) \nabla \nabla \cdot\left(\partial_{t} \boldsymbol{u}\right)-\partial_{t} \boldsymbol{j}\right) \cdot \partial_{t}^{2} \overline{\boldsymbol{u}}\right. \\
& \left.\quad+(\lambda+\mu) \nabla \cdot\left(\partial_{t}^{2} \boldsymbol{u}\right) \nabla \cdot\left(\partial_{t} \overline{\boldsymbol{u}}\right)+\mu \nabla\left(\partial_{t}^{2} \boldsymbol{u}\right): \nabla\left(\partial_{t} \overline{\boldsymbol{u}}\right)\right) \mathrm{d} \boldsymbol{x} \mathrm{d} \tau \\
= & \rho_{1} \int_{0}^{t} \int_{\Omega_{2}} \operatorname{Re}\left(-\mu \nabla\left(\partial_{t} \boldsymbol{u}\right): \nabla\left(\partial_{t}^{2} \overline{\boldsymbol{u}}\right)-(\lambda+\mu) \nabla \cdot\left(\partial_{t} \boldsymbol{u}\right) \nabla \cdot\left(\partial_{t}^{2} \overline{\boldsymbol{u}}\right)\right. \\
& \left.\quad+(\lambda+\mu) \nabla \cdot\left(\partial_{t}^{2} \boldsymbol{u}\right) \nabla \cdot\left(\partial_{t} \overline{\boldsymbol{u}}\right)+\mu \nabla\left(\partial_{t}^{2} \boldsymbol{u}\right): \nabla\left(\partial_{t} \overline{\boldsymbol{u}}\right)\right) \mathrm{d} \boldsymbol{x} \mathrm{d} \tau \\
& \quad-2 \operatorname{Re} \rho_{1} \int_{0}^{t} \int_{\Omega_{2}} \partial_{t} \boldsymbol{j} \cdot \partial_{t}^{2} \overline{\boldsymbol{u}} \mathrm{d} \boldsymbol{x} \mathrm{d} \tau+2 \operatorname{Re} \rho_{1} \int_{0}^{t} \int_{\Gamma_{f}}\left(\boldsymbol{\sigma}\left(\partial_{t} \boldsymbol{u}\right) \cdot \boldsymbol{n}\right) \cdot \partial_{t}^{2} \overline{\boldsymbol{u}} \mathrm{d} \gamma \mathrm{d} \tau \\
(3.19) \quad & 2 \operatorname{Re} \rho_{1} \int_{0}^{t} \int_{\Omega_{2}} \partial_{t} \boldsymbol{j} \cdot \partial_{t}^{2} \overline{\boldsymbol{u}} \mathrm{d} \boldsymbol{x} \mathrm{d} \tau-2 \operatorname{Re} \rho_{1} \int_{0}^{t} \int_{\Gamma_{f}} \partial_{t} p \boldsymbol{n} \cdot \partial_{t}^{2} \overline{\boldsymbol{u}} \mathrm{d} \gamma \mathrm{d} \tau .
\end{aligned}
$$


Since $\mathscr{E}(0)=0$, combining (3.17) $-(3.19)$ and (3.15) gives

$$
\begin{aligned}
\mathscr{E}(t) & =2 \operatorname{Re} \int_{0}^{t} \int_{\Gamma_{h}}(\mathscr{T} p) \partial_{t} \bar{p} \mathrm{~d} \boldsymbol{r} \mathrm{d} \tau-2 \operatorname{Re} \rho_{1} \int_{0}^{t} \int_{\Omega_{2}} \partial_{t} \boldsymbol{j} \cdot \partial_{t}^{2} \overline{\boldsymbol{u}} \mathrm{d} \boldsymbol{x} \mathrm{d} \tau \\
& \leq-2 \operatorname{Re} \rho_{1} \int_{0}^{t} \int_{\Omega_{2}} \partial_{t} \boldsymbol{j} \cdot \partial_{t}^{2} \overline{\boldsymbol{u}} \mathrm{d} \boldsymbol{x} \mathrm{d} \tau \\
& \leq 2 \rho_{1} \max _{t \in[0, T]}\left\|\partial_{t}^{2} \boldsymbol{u}\right\|_{L^{2}\left(\Omega_{2}\right)^{3}}\left\|\partial_{t} \boldsymbol{j}\right\|_{L^{1}\left(0, T ; L^{2}\left(\Omega_{2}\right)^{3}\right)} .
\end{aligned}
$$

Thus, we can obtain the estimate for the air/fluid pressure $p$ :

$$
\begin{aligned}
\max _{t \in[0, T]} & \left(\left\|\partial_{t} p\right\|_{L^{2}\left(\Omega_{1}\right)}^{2}+\|\nabla p\|_{L^{2}\left(\Omega_{1}\right)^{3}}^{2}\right) \\
& \leq \max _{t \in[0, T]}\left(\left\|\partial_{t} p\right\|_{L^{2}\left(\Omega_{1}\right)}^{2}+\|\nabla p\|_{L^{2}\left(\Omega_{1}\right)^{3}}^{2}+\left\|\partial_{t}^{2} \boldsymbol{u}\right\|_{L^{2}\left(\Omega_{2}\right)^{3}}^{2}\right) \\
& \lesssim \max _{t \in[0, T]} \mathscr{E}(t) \lesssim \max _{t \in[0, T]}\left\|\partial_{t}^{2} \boldsymbol{u}\right\|_{L^{2}\left(\Omega_{2}\right)^{3}}\left\|\partial_{t} \boldsymbol{j}\right\|_{L^{1}\left(0, T ; L^{2}\left(\Omega_{2}\right)^{3}\right)}
\end{aligned}
$$

It follows from Young's inequality that

$$
\max _{t \in[0, T]}\left(\left\|\partial_{t} p\right\|_{L^{2}\left(\Omega_{1}\right)}+\|\nabla p\|_{L^{2}\left(\Omega_{1}\right)^{3}}\right) \lesssim\left\|\partial_{t} \boldsymbol{j}\right\|_{L^{1}\left(0, T ; L^{2}\left(\Omega_{2}\right)^{3}\right)},
$$

which shows the stability estimate (3.13).

For the elastic displacement $\boldsymbol{u}$, we can also obtain

$$
\begin{aligned}
& \max _{t \in[0, T]}\left(\left\|\partial_{t}^{2} \boldsymbol{u}\right\|_{L^{2}\left(\Omega_{2}\right)^{3}}^{2}+\left\|\nabla \cdot\left(\partial_{t} \boldsymbol{u}\right)\right\|_{L^{2}\left(\Omega_{2}\right)}^{2}+\left\|\nabla\left(\partial_{t} \boldsymbol{u}\right)\right\|_{F\left(\Omega_{2}\right)}^{2}\right) \\
& \lesssim \max _{[0, T]} \mathscr{E}(t) \lesssim \max _{t \in[0, T]}\left\|\partial_{t}^{2} \boldsymbol{u}\right\|_{L^{2}\left(\Omega_{2}\right)^{3}}\left\|\partial_{t} \boldsymbol{j}\right\|_{L^{1}\left(0, T ; L^{2}\left(\Omega_{2}\right)^{3}\right)}
\end{aligned}
$$

It follows from the Cauchy-Schwarz inequality that

$$
\begin{array}{r}
\max _{t \in[0, T]}\left(\left\|\partial_{t}^{2} \boldsymbol{u}\right\|_{L^{2}\left(\Omega_{2}\right)^{3}}^{2}+\left\|\nabla \cdot\left(\partial_{t} \boldsymbol{u}\right)\right\|_{L^{2}\left(\Omega_{2}\right)}^{2}+\left\|\nabla\left(\partial_{t} \boldsymbol{u}\right)\right\|_{F\left(\Omega_{2}\right)}^{2}\right) \\
\lesssim\left\|\partial_{t} \boldsymbol{j}\right\|_{L^{1}\left(0, T ; L^{2}\left(\Omega_{2}\right)^{3}\right)}^{2} .
\end{array}
$$

For any $0<t \leq T$, using the epsilon inequality leads to

$$
\left\|\partial_{t} \boldsymbol{u}\right\|_{L^{2}\left(\Omega_{2}\right)^{3}}^{2}=\int_{0}^{t} \partial_{\tau}\left\|\partial_{\tau} \boldsymbol{u}(\cdot, \tau)\right\|_{L^{2}\left(\Omega_{2}\right)^{3}}^{2} \mathrm{~d} \tau \leq \epsilon T\left\|\partial_{t} \boldsymbol{u}\right\|_{L^{2}\left(\Omega_{2}\right)^{3}}^{2}+\frac{T}{\epsilon}\left\|\partial_{t}^{2} \boldsymbol{u}\right\|_{L^{2}\left(\Omega_{2}\right)^{3}}^{2} .
$$

Here we choose $\epsilon>0$ small enough such that $\epsilon T<1$, e.g., $\epsilon=\frac{1}{2 T}$. Hence we have

$$
\left\|\partial_{t} \boldsymbol{u}\right\|_{L^{2}\left(\Omega_{2}\right)^{3}}^{2} \lesssim\left\|\partial_{t}^{2} \boldsymbol{u}\right\|_{L^{2}\left(\Omega_{2}\right)^{3}}^{2}
$$

Similarly, we can obtain

$$
\|\nabla \cdot \boldsymbol{u}\|_{L^{2}\left(\Omega_{2}\right)}^{2} \lesssim\left\|\nabla \cdot\left(\partial_{t} \boldsymbol{u}\right)\right\|_{L^{2}\left(\Omega_{2}\right)}^{2}, \quad\|\nabla \boldsymbol{u}\|_{F\left(\Omega_{2}\right)}^{2} \lesssim\left\|\nabla\left(\partial_{t} \boldsymbol{u}\right)\right\|_{F\left(\Omega_{2}\right)}^{2} .
$$

Combining (3.20)-(3.22) gives

$$
\max _{t \in[0, T]}\left(\left\|\partial_{t} \boldsymbol{u}\right\|_{L^{2}\left(\Omega_{2}\right)^{3}}^{2}+\|\nabla \cdot \boldsymbol{u}\|_{L^{2}\left(\Omega_{2}\right)}^{2}+\|\nabla \boldsymbol{u}\|_{F\left(\Omega_{2}\right)}^{2}\right) \lesssim\left\|\partial_{t} \boldsymbol{j}\right\|_{L^{1}\left(0, T ; L^{2}\left(\Omega_{2}\right)^{3}\right)}^{2},
$$

which shows the estimate (3.14). 
3.3. A priori estimates. In what follows, we derive a priori stability estimates for the air/fluid pressure $p$ and the displacement $\boldsymbol{u}$ with a minimum regularity requirement for the data and an explicit dependence on the time.

We shall consider the elastic wave equation for $\partial_{t} \boldsymbol{u}$ in order to match the interface conditions when deducing the stability estimates. Taking the partial derivative of (3.11b) - (3.11e) and (3.11f) with respect to $t$, we obtain a new reduced problem:

$$
\begin{cases}\Delta p-\frac{1}{c^{2}} \partial_{t}^{2} p=0 & \text { in } \Omega_{1}, t>0 \\ \partial_{\boldsymbol{\nu}} p=\mathscr{T} p & \text { on } \Gamma_{h}, t>0, \\ \partial_{\boldsymbol{n}} p=-\rho_{1} \boldsymbol{n} \cdot \partial_{t}^{2} \boldsymbol{u} & \text { on } \Gamma_{f}, t>0, \\ \left.p\right|_{t=0}=\left.\partial_{t} p\right|_{t=0}=0 & \text { in } \Omega_{1} \\ \mu \Delta\left(\partial_{t} \boldsymbol{u}\right)+(\lambda+\mu) \nabla \nabla \cdot\left(\partial_{t} \boldsymbol{u}\right)-\rho_{2} \partial_{t}^{2}\left(\partial_{t} \boldsymbol{u}\right)=\partial_{t} \boldsymbol{j} & \text { in } \Omega_{2}, t>0, \\ \left.\partial_{t} \boldsymbol{u}\right|_{t=0}=0 & \text { in } \Omega_{2}, \\ \left.\partial_{t}^{2} \boldsymbol{u}\right|_{t=0}=\left.\rho_{2}^{-1}(\mu \Delta \boldsymbol{u}+(\lambda+\mu) \nabla \nabla \cdot \boldsymbol{u}-\boldsymbol{j})\right|_{t=0}=0 & \text { in } \Omega_{2}, \\ -\partial_{t} p \boldsymbol{n}=\partial_{t}(\boldsymbol{\sigma}(\boldsymbol{u})) \cdot \boldsymbol{n}=\boldsymbol{\sigma}\left(\partial_{t} \boldsymbol{u}\right) \cdot \boldsymbol{n} & \text { on } \Gamma_{f}, t>0, \\ \partial_{t} \boldsymbol{u}=0 & \text { on } \Gamma_{g}, t>0 .\end{cases}
$$

The variational problems of (3.23) is to find $(p, \boldsymbol{u}) \in H^{1}\left(\Omega_{1}\right) \times \in H_{\Gamma_{g}}^{1}\left(\Omega_{2}\right)^{3}$ for all $t>0$ such that

$$
\begin{aligned}
\int_{\Omega_{1}} \frac{1}{c^{2}} \partial_{t}^{2} p \bar{q} \mathrm{~d} \boldsymbol{x} & =-\int_{\Omega_{1}} \nabla p \cdot \nabla \bar{q} \mathrm{~d} \boldsymbol{x}+\int_{\Gamma_{h}}(\mathscr{T} p) \bar{q} \mathrm{~d} \boldsymbol{r}-\int_{\Gamma_{f}} \partial_{\boldsymbol{n}} p \bar{q} \mathrm{~d} \gamma \\
(3.24) \quad & =-\int_{\Omega_{1}} \nabla p \cdot \nabla \bar{q} \mathrm{~d} \boldsymbol{x}+\int_{\Gamma_{h}}(\mathscr{T} p) \bar{q} \mathrm{~d} \boldsymbol{r}+\int_{\Gamma_{f}} \rho_{1}\left(\boldsymbol{n} \cdot \partial_{t}^{2} \boldsymbol{u}\right) \bar{q} \mathrm{~d} \gamma, \quad \forall q \in H^{1}\left(\Omega_{1}\right)
\end{aligned}
$$

and

$$
\begin{aligned}
\int_{\Omega_{2}} \rho_{2} \partial_{t}^{2}\left(\partial_{t} \boldsymbol{u}\right) \cdot \overline{\boldsymbol{v}} \mathrm{d} \boldsymbol{x}=- & \int_{\Omega_{2}}\left(\mu \nabla\left(\partial_{t} \boldsymbol{u}\right): \nabla \overline{\boldsymbol{v}}+(\lambda+\mu)\left(\nabla \cdot\left(\partial_{t} \boldsymbol{u}\right)\right)(\nabla \cdot \overline{\boldsymbol{v}})\right) \mathrm{d} \boldsymbol{x} \\
& -\int_{\Omega_{2}} \partial_{t} \boldsymbol{j} \cdot \overline{\boldsymbol{v}} \mathrm{d} \boldsymbol{x}+\int_{\Gamma_{f}}\left(\boldsymbol{\sigma}\left(\partial_{t} \boldsymbol{u}\right) \cdot \boldsymbol{n}\right) \cdot \overline{\boldsymbol{v}} \mathrm{d} \gamma \\
=- & \int_{\Omega_{2}}\left(\mu \nabla\left(\partial_{t} \boldsymbol{u}\right): \nabla \overline{\boldsymbol{v}}+(\lambda+\mu)\left(\nabla \cdot\left(\partial_{t} \boldsymbol{u}\right)\right)(\nabla \cdot \overline{\boldsymbol{v}})+\left(\partial_{t} \boldsymbol{j}\right) \cdot \overline{\boldsymbol{v}}\right) \mathrm{d} \boldsymbol{x} \\
& -\int_{\Gamma_{f}}\left(\partial_{t} p\right)(\boldsymbol{n} \cdot \overline{\boldsymbol{v}}) \mathrm{d} \gamma, \quad \forall \boldsymbol{v} \in H_{\Gamma_{g}}^{1}\left(\Omega_{2}\right)^{3} .
\end{aligned}
$$

To show the stability of the solution, we follow the argument in 34 but with a careful study of the TBC. The following lemma is useful for the subsequent analysis.

Lemma 3.3. Given $\xi \geq 0$ and $p \in H^{1}\left(\Omega_{1}\right)$, we have

$$
\operatorname{Re} \int_{\Gamma_{h}} \int_{0}^{\xi}\left(\int_{0}^{t} \mathscr{T} p(\cdot, \tau) \mathrm{d} \tau\right) \bar{p}(\cdot, t) \mathrm{d} t \mathrm{~d} \boldsymbol{r} \leq 0 .
$$

Proof. Let $\tilde{p}$ be the extension of $p$ with respect to $t$ in $\mathbb{R}$ such that $\tilde{p}=0$ outside 
the interval $[0, \xi]$. We obtain from the Parseval identity [2.6) and Lemma 2.6 that

$$
\begin{aligned}
\operatorname{Re} \int_{\Gamma_{h}} & \int_{0}^{\xi} e^{-2 s_{1} t}\left(\int_{0}^{\tau} \mathscr{T} p(\cdot, \tau) \mathrm{d} \tau\right) \bar{p}(\cdot, t) \mathrm{d} t \mathrm{~d} \boldsymbol{r} \\
& =\operatorname{Re} \int_{\Gamma_{h}} \int_{0}^{\infty} e^{-2 s_{1} t}\left(\int_{0}^{t} \mathscr{T} \tilde{p}(\cdot, \tau) \mathrm{d} \tau\right) \overline{\tilde{p}}(\cdot, t) \mathrm{d} t \mathrm{~d} \boldsymbol{r} \\
& =\operatorname{Re} \int_{\Gamma_{h}} \int_{0}^{\infty} e^{-2 s_{1} t}\left(\int_{0}^{t} \mathscr{L}^{-1} \circ \mathscr{B} \circ \mathscr{L} \tilde{p}(\cdot, \tau) \mathrm{d} \tau\right) \overline{\tilde{p}}(\cdot, t) \mathrm{d} t \mathrm{~d} \boldsymbol{r} \\
& =\operatorname{Re} \int_{\Gamma_{h}} \int_{0}^{\infty} e^{-2 s_{1} t}\left(\mathscr{L}^{-1} \circ\left(s^{-1} \mathscr{B}\right) \circ \mathscr{L} \tilde{p}(\cdot, t) \overline{\tilde{p}}(\cdot, t)\right) \mathrm{d} t \mathrm{~d} \boldsymbol{r} \\
& =\frac{1}{2 \pi} \int_{-\infty}^{\infty} \operatorname{Re} \int_{\Gamma_{h}} s^{-1} \mathscr{B} \breve{p}(\cdot, s) \overline{\tilde{p}}(\cdot, s) \mathrm{d} \boldsymbol{r} \mathrm{d} s_{2} \\
& =\frac{1}{2 \pi} \int_{-\infty}^{\infty} \operatorname{Re}\left\langle s^{-1} \mathscr{B} \breve{p}, \breve{p}\right\rangle_{\Gamma_{h}} \mathrm{~d} s_{2} \leq 0,
\end{aligned}
$$

where we have used the fact that

$$
\int_{0}^{t} p(\cdot, \tau) \mathrm{d} \tau=\mathscr{L}^{-1}\left(s^{-1} \breve{p}(\cdot, s)\right)
$$

The proof is completed after taking the limit $s_{1} \rightarrow 0$. $\mathbf{Q}$

Theorem 3.4. Let $(p, \boldsymbol{u}) \in H^{1}\left(\Omega_{1}\right) \times H_{\Gamma_{g}}^{1}\left(\Omega_{2}\right)^{3}$ be the solution of (3.24)-(3.25).

Given $\partial_{t} \boldsymbol{j} \in L^{1}\left(0, T ; L^{2}\left(\Omega_{2}\right)^{3}\right)$, for any $T>0$, we have

$$
\begin{aligned}
\|p\|_{L^{\infty}\left(0, T ; L^{2}\left(\Omega_{1}\right)\right)} & \lesssim T\left\|\partial_{t} \boldsymbol{j}\right\|_{L^{1}\left(0, T ; L^{2}\left(\Omega_{2}\right)^{3}\right)} \\
\|\boldsymbol{u}\|_{L^{\infty}\left(0, T ; L^{2}\left(\Omega_{2}\right)^{3}\right)} & \lesssim T^{2}\left\|\partial_{t} \boldsymbol{j}\right\|_{L^{1}\left(0, T ; L^{2}\left(\Omega_{2}\right)^{3}\right)} \\
\|p\|_{L^{2}\left(0, T ; L^{2}\left(\Omega_{1}\right)\right)} & \lesssim T^{3 / 2}\left\|\partial_{t} \boldsymbol{j}\right\|_{L^{1}\left(0, T ; L^{2}\left(\Omega_{2}\right)^{3}\right)} \\
\|\boldsymbol{u}\|_{L^{2}\left(0, T ; L^{2}\left(\Omega_{2}\right)^{3}\right)} & \lesssim T^{5 / 2}\left\|\partial_{t} \boldsymbol{j}\right\|_{L^{1}\left(0, T ; L^{2}\left(\Omega_{2}\right)^{3}\right)}
\end{aligned}
$$

Proof. Let $0<\theta<T$ and define an auxiliary function

$$
\psi_{1}(\boldsymbol{x}, t)=\int_{t}^{\theta} p(\boldsymbol{x}, \tau) \mathrm{d} \tau, \quad \boldsymbol{x} \in \Omega_{1}, 0 \leq t \leq \theta .
$$

It is clear to note that

$$
\psi_{1}(\boldsymbol{x}, \theta)=0, \quad \partial_{t} \psi_{1}(\boldsymbol{x}, t)=-p(\boldsymbol{x}, t) .
$$

For any $\phi(\boldsymbol{x}, t) \in L^{2}\left(0, \xi ; L^{2}\left(\Omega_{1}\right)\right)$, we have

$$
\int_{0}^{\theta} \phi(\boldsymbol{x}, t) \bar{\psi}_{1}(\boldsymbol{x}, t) \mathrm{d} t=\int_{0}^{\theta}\left(\int_{0}^{t} \phi(\boldsymbol{x}, \tau) \mathrm{d} \tau\right) \bar{p}(\boldsymbol{x}, t) \mathrm{d} t
$$


Indeed, we have from the integration by parts and (3.30) that

$$
\begin{aligned}
\int_{0}^{\theta} & \phi(\boldsymbol{x}, t) \bar{\psi}_{1}(\boldsymbol{x}, t) \mathrm{d} t=\int_{0}^{\theta}\left(\phi(\boldsymbol{x}, t) \int_{t}^{\theta} \bar{p}(\boldsymbol{x}, \tau) \mathrm{d} \tau\right) \mathrm{d} t \\
& =\int_{0}^{\theta} \int_{t}^{\theta} \bar{p}(\boldsymbol{x}, \tau) \mathrm{d} \tau \mathrm{d}\left(\int_{0}^{t} \phi(\boldsymbol{x}, \varsigma) \mathrm{d} \varsigma\right) \\
& =\left.\int_{t}^{\theta} \bar{p}(\boldsymbol{x}, \tau) \mathrm{d} \tau \int_{0}^{t} \phi(\boldsymbol{x}, \varsigma) \mathrm{d} \varsigma\right|_{0} ^{\theta}+\int_{0}^{\theta}\left(\int_{0}^{t} \phi(\boldsymbol{x}, \varsigma) \mathrm{d} \varsigma\right) \bar{p}(\boldsymbol{x}, t) \mathrm{d} t \\
& =\int_{0}^{\theta}\left(\int_{0}^{t} \phi(\boldsymbol{x}, \tau) \mathrm{d} \tau\right) \bar{p}(\boldsymbol{x}, t) \mathrm{d} t .
\end{aligned}
$$

Next, we take the test function $q=\psi_{1}$ in (3.24) and get

$$
\begin{aligned}
\int_{\Omega_{1}} \frac{1}{c^{2}} \partial_{t}^{2} p \bar{\psi}_{1} \mathrm{~d} \boldsymbol{x}=-\int_{\Omega_{1}} \nabla p \cdot \nabla \bar{\psi}_{1} \mathrm{~d} \boldsymbol{x}+\int_{\Gamma_{h}}(\mathscr{T} p) \bar{\psi}_{1} \mathrm{~d} \boldsymbol{r} \\
+\int_{\Gamma_{f}} \rho_{1}\left(\boldsymbol{n} \cdot \partial_{t}^{2} \boldsymbol{u}\right) \bar{\psi}_{1} \mathrm{~d} \gamma
\end{aligned}
$$

It follows from (3.30) and the initial conditions (3.11c) that

$$
\begin{aligned}
\operatorname{Re} \int_{0}^{\theta} \int_{\Omega_{1}} \frac{1}{c^{2}} \partial_{t}^{2} p \bar{\psi}_{1} \mathrm{~d} \boldsymbol{x} \mathrm{d} t & =\operatorname{Re} \int_{\Omega_{1}} \int_{0}^{\theta} \frac{1}{c^{2}}\left(\partial_{t}\left(\partial_{t} p \bar{\psi}_{1}\right)+\partial_{t} p \bar{p}\right) \mathrm{d} t \mathrm{~d} \boldsymbol{x} \\
& =\operatorname{Re} \int_{\Omega_{1}} \frac{1}{c^{2}}\left(\left.\partial_{t} p \bar{\psi}_{1}\right|_{0} ^{\theta}+\left.\frac{1}{2}|p|^{2}\right|_{0} ^{\theta}\right) \mathrm{d} \boldsymbol{x} \\
& =\frac{1}{2}\left\|\frac{1}{c} p(\cdot, \theta)\right\|_{L^{2}\left(\Omega_{1}\right)}^{2}
\end{aligned}
$$

It is easy to verify that

$$
\begin{aligned}
\operatorname{Re} \int_{0}^{\theta} & \int_{\Gamma_{f}} \rho_{1}\left(\boldsymbol{n} \cdot \partial_{t}^{2} \boldsymbol{u}\right) \bar{\psi}_{1} \mathrm{~d} \gamma \mathrm{d} t=\operatorname{Re} \int_{\Gamma_{f}} \int_{0}^{\theta} \rho_{1}\left(\partial_{t}\left(\boldsymbol{n} \cdot \partial_{t} \boldsymbol{u} \bar{\psi}_{1}\right)+\left(\boldsymbol{n} \cdot \partial_{t} \boldsymbol{u}\right) \bar{p}\right) \mathrm{d} t \mathrm{~d} \gamma \\
& =\operatorname{Re} \int_{\Gamma_{f}} \rho_{1}\left(\left.\boldsymbol{n} \cdot \partial_{t} \boldsymbol{u} \bar{\psi}_{1}\right|_{0} ^{\theta}\right) \mathrm{d} \gamma+\operatorname{Re} \int_{0}^{\theta} \int_{\Gamma_{f}} \rho_{1}\left(\boldsymbol{n} \cdot \partial_{t} \boldsymbol{u}\right) \bar{p} \mathrm{~d} \gamma \mathrm{d} t \\
& =\operatorname{Re} \int_{0}^{\theta} \int_{\Gamma_{f}} \rho_{1}\left(\boldsymbol{n} \cdot \partial_{t} \boldsymbol{u}\right) \bar{p} \mathrm{~d} \gamma \mathrm{d} t
\end{aligned}
$$

Integrating (3.32) from $t=0$ to $t=\theta$ and taking the real parts yield

$$
\begin{aligned}
\frac{1}{2} \| & \frac{1}{c} p(\cdot, \theta) \|_{L^{2}\left(\Omega_{1}\right)}^{2}+\operatorname{Re} \int_{0}^{\theta} \int_{\Omega_{1}} \nabla p \cdot \bar{\psi}_{1} \mathrm{~d} \boldsymbol{x} \mathrm{d} t \\
& =\frac{1}{2}\left\|\frac{1}{c} p(\cdot, \theta)\right\|_{L^{2}\left(\Omega_{1}\right)}^{2}+\frac{1}{2} \int_{\Omega_{1}}\left|\int_{0}^{\theta} \nabla p(\cdot, t) \mathrm{d} t\right|^{2} \mathrm{~d} \boldsymbol{x} \\
& =\operatorname{Re} \int_{0}^{\theta}\left\langle\mathscr{T} p, \psi_{1}\right\rangle_{\Gamma_{h}} \mathrm{~d} t+\operatorname{Re} \int_{0}^{\theta} \int_{\Gamma_{f}} \rho_{1}\left(\boldsymbol{n} \cdot \partial_{t}^{2} \boldsymbol{u}\right) \bar{\psi} \mathrm{d} \gamma \mathrm{d} t \\
& =\operatorname{Re} \int_{0}^{\theta}\left\langle\mathscr{T} p, \psi_{1}\right\rangle_{\Gamma_{h}} \mathrm{~d} t+\operatorname{Re} \int_{0}^{\theta} \int_{\Gamma_{f}} \rho_{1}\left(\boldsymbol{n} \cdot \partial_{t} \boldsymbol{u}\right) \bar{p} \mathrm{~d} \gamma \mathrm{d} t .
\end{aligned}
$$


We define another auxiliary function

$$
\boldsymbol{\psi}_{2}(\boldsymbol{x}, t)=\int_{t}^{\theta} \partial_{\tau} \boldsymbol{u}(\boldsymbol{x}, \tau) \mathrm{d} \tau, \quad \boldsymbol{x} \in \Omega_{2}, 0 \leq t \leq \theta<T .
$$

Clearly, we have

$$
\boldsymbol{\psi}_{2}(\boldsymbol{x}, \theta)=0, \quad \partial_{t} \boldsymbol{\psi}_{2}(\boldsymbol{x}, t)=-\partial_{t} \boldsymbol{u}(\boldsymbol{x}, t) .
$$

Using the similar proof as that for (3.31), for any $\phi(x, t) \in L^{2}\left(0, \xi ; L^{2}\left(\Omega_{2}\right)^{2}\right)$, we may show that

$$
\int_{0}^{\theta} \phi(\boldsymbol{x}, t) \cdot \overline{\boldsymbol{\psi}}_{2}(\boldsymbol{x}, t) \mathrm{d} t=\int_{0}^{\theta}\left(\int_{0}^{t} \phi(\boldsymbol{x}, \tau) \mathrm{d} \tau\right) \cdot \partial_{t} \overline{\boldsymbol{u}}(\boldsymbol{x}, t) \mathrm{d} t .
$$

Taking the test function $\boldsymbol{v}=\boldsymbol{\psi}_{2}$ in (3.25), we can get

$$
\begin{array}{r}
\int_{\Omega_{2}} \rho_{2} \partial_{t}^{2}\left(\partial_{t} \boldsymbol{u}\right) \cdot \overline{\boldsymbol{\psi}}_{2} \mathrm{~d} \boldsymbol{x}=-\int_{\Omega_{2}}\left(\mu \nabla\left(\partial_{t} \boldsymbol{u}\right): \nabla \overline{\boldsymbol{\psi}}_{2}+(\lambda+\mu)\left(\nabla \cdot\left(\partial_{t} \boldsymbol{u}\right)\right)\left(\nabla \cdot \overline{\boldsymbol{\psi}}_{2}\right)\right. \\
\left.+\partial_{t} \boldsymbol{j} \cdot \overline{\boldsymbol{\psi}}_{2}\right) \mathrm{d} \boldsymbol{x}-\int_{\Gamma_{f}}\left(\partial_{t} p\right)\left(\boldsymbol{n} \cdot \overline{\boldsymbol{\psi}}_{2}\right) \mathrm{d} \gamma
\end{array}
$$

It follows from (3.34) and the initial condition in (3.23) that

$$
\begin{aligned}
\operatorname{Re} \int_{0}^{\theta} \int_{\Omega_{2}} \rho_{2} \partial_{t}^{2}\left(\partial_{t} \boldsymbol{u}\right) \cdot \overline{\boldsymbol{\psi}}_{2} \mathrm{~d} \boldsymbol{x} \mathrm{d} t & =\operatorname{Re} \int_{\Omega_{2}} \int_{0}^{\theta} \rho_{2}\left(\partial_{t}\left(\partial_{t}^{2} \boldsymbol{u} \cdot \overline{\boldsymbol{\psi}}_{2}\right)+\partial_{t}^{2} \boldsymbol{u} \cdot \partial_{t} \overline{\boldsymbol{u}}\right) \mathrm{d} t \mathrm{~d} \boldsymbol{x} \\
& =\operatorname{Re} \int_{\Omega_{2}} \rho_{2}\left(\left.\left(\partial_{t}^{2} \boldsymbol{u} \cdot \overline{\boldsymbol{\psi}}_{2}\right)\right|_{0} ^{\theta}+\left.\frac{1}{2}\left|\partial_{t} \boldsymbol{u}\right|^{2}\right|_{0} ^{\theta}\right) \mathrm{d} \boldsymbol{x} \\
& =\frac{\rho_{2}}{2}\left\|\partial_{t} \boldsymbol{u}(\cdot, \theta)\right\|_{L^{2}\left(\Omega_{2}\right)^{3}}^{2}
\end{aligned}
$$

and

$$
\begin{aligned}
\operatorname{Re} \int_{0}^{\theta} \int_{\Gamma_{f}}\left(\partial_{t} p\right)\left(\boldsymbol{n} \cdot \overline{\boldsymbol{\psi}}_{2}\right) \mathrm{d} \gamma \mathrm{d} t & =\operatorname{Re} \int_{\Gamma_{f}} \int_{0}^{\theta}\left(\partial_{t}\left(p \boldsymbol{n} \cdot \overline{\boldsymbol{\psi}}_{2}\right)+p\left(\boldsymbol{n} \cdot \partial_{t} \overline{\boldsymbol{u}}\right)\right) \mathrm{d} t \mathrm{~d} \gamma \\
& =\left.\operatorname{Re} \int_{\Gamma_{f}}\left(p \boldsymbol{n} \cdot \overline{\boldsymbol{\psi}}_{2}\right)\right|_{0} ^{\theta} \mathrm{d} \gamma+\operatorname{Re} \int_{0}^{\theta} \int_{\Gamma_{f}} p\left(\boldsymbol{n} \cdot \partial_{t} \overline{\boldsymbol{u}}\right) \mathrm{d} \gamma \mathrm{d} t \\
& =\operatorname{Re} \int_{0}^{\theta} \int_{\Gamma_{f}} p\left(\boldsymbol{n} \cdot \partial_{t} \overline{\boldsymbol{u}}\right) \mathrm{d} \gamma \mathrm{d} t .
\end{aligned}
$$

Integrating (3.36) from $t=0$ to $t=\theta$ and taking the real parts yield

$$
\begin{aligned}
& \frac{\rho_{2}}{2}\left\|\partial_{t} \boldsymbol{u}(\cdot, \theta)\right\|_{L^{2}\left(\Omega_{2}\right)^{3}}^{2}+\operatorname{Re} \int_{0}^{\theta} \int_{\Omega_{2}}\left(\mu \nabla\left(\partial_{t} \boldsymbol{u}(\cdot, t)\right): \nabla \overline{\boldsymbol{\psi}}_{2}(\cdot, t)\right. \\
& \left.\quad+(\lambda+\mu)\left(\nabla \cdot\left(\partial_{t} \boldsymbol{u}(\cdot, t)\right)\right)\left(\nabla \cdot \overline{\boldsymbol{\psi}}_{2}(\cdot, t)\right)\right) \mathrm{d} \boldsymbol{x} \mathrm{d} t \\
& =\frac{\rho_{2}}{2}\left\|\partial_{t} \boldsymbol{u}(\cdot, \theta)\right\|_{L^{2}\left(\Omega_{2}\right)^{2}}^{2}+\frac{1}{2} \int_{\Omega_{2}}\left(\mu\left|\int_{0}^{\theta} \nabla\left(\partial_{t} \boldsymbol{u}(\cdot, t)\right) \mathrm{d} t\right|_{F}^{2}\right. \\
& \left.\quad+(\lambda+\mu)\left|\int_{0}^{\theta} \nabla \cdot\left(\partial_{t} \boldsymbol{u}(\cdot, t)\right) \mathrm{d} t\right|^{2}\right) \mathrm{d} \boldsymbol{x} \\
& =-\operatorname{Re} \int_{0}^{\theta} \int_{\Omega_{2}} \partial_{t} \boldsymbol{j} \cdot \overline{\boldsymbol{\psi}}_{2} \mathrm{~d} \boldsymbol{x} \mathrm{d} t-\operatorname{Re} \int_{0}^{\theta} \int_{\Gamma_{f}} p\left(\boldsymbol{n} \cdot \partial_{t} \overline{\boldsymbol{u}}\right) \mathrm{d} \gamma \mathrm{d} t,
\end{aligned}
$$


where

$$
\left|\int_{0}^{\theta} \nabla\left(\partial_{t} \boldsymbol{u}(\cdot, t)\right) \mathrm{d} t\right|_{F}^{2}:=\int_{0}^{\theta} \nabla\left(\partial_{t} \boldsymbol{u}(\cdot, t)\right) \mathrm{d} t: \int_{0}^{\theta} \nabla\left(\partial_{t} \overline{\boldsymbol{u}}(\cdot, t)\right) \mathrm{d} t
$$

Multiplying (3.37) by $\rho_{1}$ and adding it to (3.33) give

$$
\begin{aligned}
& \frac{1}{2}\left\|\frac{1}{c} p(\cdot, \theta)\right\|_{L^{2}\left(\Omega_{1}\right)}^{2}+\frac{1}{2} \int_{\Omega_{1}}\left|\int_{0}^{\theta} \nabla p(\cdot, t) \mathrm{d} t\right|^{2} \mathrm{~d} \boldsymbol{x}+\frac{\rho_{1} \rho_{2}}{2}\left\|\partial_{t} \boldsymbol{u}(\cdot, \theta)\right\|_{L^{2}\left(\Omega_{2}\right)^{3}}^{2} \\
& \quad+\frac{\rho_{1}}{2} \int_{\Omega_{2}}\left(\mu\left|\int_{0}^{\theta} \nabla\left(\partial_{t} \boldsymbol{u}(\cdot, t)\right) \mathrm{d} t\right|_{F}^{2}+(\lambda+\mu)\left|\int_{0}^{\theta} \nabla \cdot\left(\partial_{t} \boldsymbol{u}(\cdot, t)\right) \mathrm{d} t\right|^{2}\right) \mathrm{d} \boldsymbol{x} \\
& =\operatorname{Re} \int_{0}^{\theta}\left\langle\mathscr{T} p, \psi_{1}\right\rangle_{\Gamma_{h}} \mathrm{~d} t+\operatorname{Re} \int_{0}^{\theta} \int_{\Gamma_{f}} \rho_{1}\left(\boldsymbol{n} \cdot \partial_{t} \boldsymbol{u}\right) \bar{p} \mathrm{~d} \gamma \mathrm{d} t \\
& \quad-\operatorname{Re} \int_{0}^{\theta} \int_{\Omega_{2}} \rho_{1}\left(\partial_{t} \boldsymbol{j} \cdot \overline{\boldsymbol{\psi}}_{2}\right) \mathrm{d} \boldsymbol{x} \mathrm{d} t-\operatorname{Re} \int_{0}^{\theta} \int_{\Gamma_{f}} \rho_{1} p\left(\boldsymbol{n} \cdot \partial_{t} \overline{\boldsymbol{u}}\right) \mathrm{d} \gamma \mathrm{d} t \\
& =\operatorname{Re} \int_{0}^{\theta}\left\langle\mathscr{T} p, \psi_{1}\right\rangle_{\Gamma_{h}} \mathrm{~d} t-\operatorname{Re} \int_{0}^{\theta} \int_{\Omega_{2}} \rho_{1}\left(\partial_{t} \boldsymbol{j} \cdot \overline{\boldsymbol{\psi}}_{2}\right) \mathrm{d} \boldsymbol{x} \mathrm{d} t .
\end{aligned}
$$

In what follows, we estimate the two terms on the right-hand side of (3.38) separately. Using Lemma 3.3 and (3.31), we obtain

$$
\begin{aligned}
\operatorname{Re} \int_{0}^{\theta}\left\langle\mathscr{T} p, \psi_{1}\right\rangle_{\Gamma_{h}} \mathrm{~d} t & =\operatorname{Re} \int_{0}^{\theta} \int_{\Gamma_{h}}(\mathscr{T} p) \bar{\psi}_{1} \mathrm{~d} \boldsymbol{r} \mathrm{d} t \\
& =\operatorname{Re} \int_{\Gamma_{h}} \int_{0}^{\theta}\left(\int_{0}^{t} \mathscr{T} p(\cdot, \tau) \mathrm{d} \tau\right) \bar{p}(\cdot, t) \mathrm{d} t \mathrm{~d} \boldsymbol{r} \leq 0 .
\end{aligned}
$$

For $0 \leq t \leq \theta \leq T$, we have from (3.35) that

$$
\begin{aligned}
\operatorname{Re} \int_{0}^{\theta} \int_{\Omega_{2}} & -\rho_{1}\left(\partial_{t} \boldsymbol{j} \cdot \overline{\boldsymbol{\psi}}_{2}\right) \mathrm{d} \boldsymbol{x} \mathrm{d} t=\rho_{1} \operatorname{Re} \int_{\Omega_{2}} \int_{0}^{\theta}\left(\int_{0}^{t}-\partial_{t} \boldsymbol{j}(\cdot, \tau) \mathrm{d} \tau\right) \cdot \partial_{t} \overline{\boldsymbol{u}}(\cdot, t) \mathrm{d} t \mathrm{~d} \boldsymbol{x} \\
& =\rho_{1} \operatorname{Re} \int_{0}^{\theta} \int_{0}^{t} \int_{\Omega_{2}}-\partial_{t} \boldsymbol{j}(\cdot, \tau) \cdot \partial_{t} \overline{\boldsymbol{u}}(\cdot, t) \mathrm{d} \boldsymbol{x} \mathrm{d} \tau \mathrm{d} t \\
& \leq \rho_{1} \int_{0}^{\theta}\left(\int_{0}^{t}\left\|\partial_{t} \boldsymbol{j}(\cdot, \tau)\right\|_{L^{2}\left(\Omega_{2}\right)^{3}} \mathrm{~d} \tau\right)\left\|\partial_{t} \boldsymbol{u}(\cdot, t)\right\|_{L^{2}\left(\Omega_{2}\right)^{2}} \mathrm{~d} t \\
& \leq \rho_{1} \int_{0}^{\theta}\left(\int_{0}^{\theta}\left\|\partial_{t} \boldsymbol{j}(\cdot, t)\right\|_{L^{2}\left(\Omega_{2}\right)^{3}} \mathrm{~d} t\right)\left\|\partial_{t} \boldsymbol{u}(\cdot, t)\right\|_{L^{2}\left(\Omega_{2}\right)^{3}} \mathrm{~d} t \\
& \leq \rho_{1}\left(\int_{0}^{\theta}\left\|\partial_{t} \boldsymbol{j}(\cdot, t)\right\|_{L^{2}\left(\Omega_{2}\right)^{3}} \mathrm{~d} t\right)\left(\int_{0}^{\theta}\left\|\partial_{t} \boldsymbol{u}(\cdot, t)\right\|_{L^{2}\left(\Omega_{2}\right)^{3}} \mathrm{~d} t\right) .
\end{aligned}
$$


Substituting (3.39) and (3.40) into (3.38), we have for any $\theta \in[0, T]$ that

$$
\begin{aligned}
& \frac{1}{2}\left\|\frac{1}{c} p(\cdot, \theta)\right\|_{L^{2}\left(\Omega_{1}\right)}^{2}+\frac{\rho_{1} \rho_{2}}{2}\left\|\partial_{t} \boldsymbol{u}(\cdot, \theta)\right\|_{L^{2}\left(\Omega_{2}\right)^{3}}^{2} \\
& \leq \frac{1}{2}\left\|\frac{1}{c} p(\cdot, \theta)\right\|_{L^{2}\left(\Omega_{1}\right)}^{2}+\frac{1}{2} \int_{\Omega_{1}}\left|\int_{0}^{\theta} \nabla p(\cdot, t) \mathrm{d} t\right|^{2} \mathrm{~d} \boldsymbol{x}+\frac{\rho_{1} \rho_{2}}{2}\left\|\partial_{t} \boldsymbol{u}(\cdot, \theta)\right\|_{L^{2}\left(\Omega_{2}\right)^{3}}^{2} \\
& \quad+\frac{\rho_{1}}{2} \int_{\Omega_{2}}\left(\mu\left|\int_{0}^{\theta} \nabla\left(\partial_{t} \boldsymbol{u}(\cdot, t)\right) \mathrm{d} t\right|_{F}^{2}+(\lambda+\mu)\left|\int_{0}^{\theta} \nabla \cdot\left(\partial_{t} \boldsymbol{u}(\cdot, t)\right) \mathrm{d} t\right|^{2}\right) \mathrm{d} \boldsymbol{x} \\
& \leq \rho_{1}\left(\int_{0}^{\theta}\left\|\partial_{t} \boldsymbol{j}(\cdot, t)\right\|_{L^{2}\left(\Omega_{2}\right)^{3}} \mathrm{~d} t\right)\left(\int_{0}^{\theta}\left\|\partial_{t} \boldsymbol{u}(\cdot, t)\right\|_{L^{2}\left(\Omega_{2}\right)^{2}} \mathrm{~d} t\right) .
\end{aligned}
$$

Taking the $L^{\infty}$ norm with respect to $\theta$ on both sides of (3.41) yields

$$
\begin{aligned}
\|p\|_{L^{\infty}\left(0, T ; L^{2}\left(\Omega_{1}\right)\right)}^{2} & +\left\|\partial_{t} \boldsymbol{u}\right\|_{L^{\infty}\left(0, T ; L^{2}\left(\Omega_{2}\right)^{3}\right)}^{2} \\
& \lesssim T\left(\left\|\partial_{t} \boldsymbol{j}\right\|_{L^{1}\left(0, T ; L^{2}\left(\Omega_{2}\right)^{3}\right)}\left\|\partial_{t} \boldsymbol{u}\right\|_{L^{\infty}\left(0, T ; L^{2}\left(\Omega_{2}\right)^{3}\right)}\right)
\end{aligned}
$$

Applying the Young inequality to the above inequality, we get

$$
\|p\|_{L^{\infty}\left(0, T ; L^{2}\left(\Omega_{1}\right)\right)}^{2}+\left\|\partial_{t} \boldsymbol{u}\right\|_{L^{\infty}\left(0, T ; L^{2}\left(\Omega_{2}\right)^{3}\right)}^{2} \lesssim T^{2}\left\|\partial_{t} \boldsymbol{j}\right\|_{L^{1}\left(0, T ; L^{2}\left(\Omega_{2}\right)^{3}\right)}^{2} .
$$

It follows from the Cauchy-Schwarz inequality that

$$
\begin{aligned}
\|p\|_{L^{\infty}\left(0, T ; L^{2}\left(\Omega_{1}\right)\right)} & \leq\|p\|_{L^{\infty}\left(0, T ; L^{2}\left(\Omega_{1}\right)\right)}+\left\|\partial_{t} \boldsymbol{u}\right\|_{L^{\infty}\left(0, T ; L^{2}\left(\Omega_{2}\right)^{3}\right)} \\
& \lesssim T\left\|\partial_{t} \boldsymbol{j}\right\|_{L^{1}\left(0, T ; L^{2}\left(\Omega_{2}\right)^{3}\right)}
\end{aligned}
$$

which gives the estimate (3.26).

For the elastic displacement $\boldsymbol{u}$, using the epsilon inequality gives

$$
\begin{aligned}
\|\boldsymbol{u}\|_{L^{\infty}\left(0, T ; L^{2}\left(\Omega_{2}\right)^{3}\right)}^{2} & =\int_{0}^{t} \partial_{\tau}\|\boldsymbol{u}(\cdot, \tau)\|_{L^{\infty}\left(0, T ; L^{2}\left(\Omega_{2}\right)^{3}\right)}^{2} \mathrm{~d} \tau \\
& \leq \epsilon T\|\boldsymbol{u}\|_{L^{\infty}\left(0, T ; L^{2}\left(\Omega_{2}\right)^{3}\right)}^{2}+\frac{T}{\epsilon}\left\|\partial_{t} \boldsymbol{u}\right\|_{L^{\infty}\left(0, T ; L^{2}\left(\Omega_{2}\right)^{3}\right)^{2}}^{2}
\end{aligned}
$$

Choosing $\epsilon=\frac{1}{2 T}$, we have from (3.42) that

$$
\begin{aligned}
\|\boldsymbol{u}\|_{L^{\infty}\left(0, T ; L^{2}\left(\Omega_{2}\right)^{3}\right)}^{2} & \lesssim T^{2}\left\|\partial_{t} \boldsymbol{u}\right\|_{L^{\infty}\left(0, T ; L^{2}\left(\Omega_{2}\right)^{3}\right)}^{2} \\
& \lesssim T^{2}\left(\|p\|_{L^{\infty}\left(0, T ; L^{2}\left(\Omega_{1}\right)\right)}^{2}+\left\|\partial_{t} \boldsymbol{u}\right\|_{L^{\infty}\left(0, T ; L^{2}\left(\Omega_{2}\right)^{3}\right)}^{2}\right) \\
& \lesssim T^{4}\left\|\partial_{t} \boldsymbol{j}\right\|_{L^{1}\left(0, T ; L^{2}\left(\Omega_{2}\right)^{3}\right)}^{2},
\end{aligned}
$$

which implies the estimate (3.27).

Integrating (3.41) with respect to $\theta$ from 0 to $T$ and using the Cauchy-Schwarz inequality, we obtain

$$
\begin{aligned}
\|p\|_{L^{2}\left(0, T ; L^{2}\left(\Omega_{1}\right)\right)}^{2} & +\left\|\partial_{t} \boldsymbol{u}\right\|_{L^{2}\left(0, T ; L^{2}\left(\Omega_{2}\right)^{3}\right)}^{2} \\
& \lesssim T^{3 / 2}\left(\left\|\partial_{t} \boldsymbol{j}\right\|_{L^{1}\left(0, T ; L^{2}\left(\Omega_{2}\right)^{3}\right)}\left\|\partial_{t} \boldsymbol{u}\right\|_{L^{2}\left(0, T ; L^{2}\left(\Omega_{2}\right)^{3}\right)}\right) .
\end{aligned}
$$

Using Young's inequality again to the above equation yields

$$
\|p\|_{L^{2}\left(0, T ; L^{2}\left(\Omega_{1}\right)\right)}^{2}+\left\|\partial_{t} \boldsymbol{u}\right\|_{L^{2}\left(0, T ; L^{2}\left(\Omega_{2}\right)^{3}\right)}^{2} \lesssim T^{3}\left\|\partial_{t} \boldsymbol{j}\right\|_{L^{1}\left(0, T ; L^{2}\left(\Omega_{2}\right)^{3}\right)}^{2} .
$$


It follows from the Cauchy-Schwarz inequality that

$$
\begin{aligned}
\|p\|_{L^{2}\left(0, T ; L^{2}\left(\Omega_{1}\right)\right)} & \leq\|p\|_{L^{2}\left(0, T ; L^{2}\left(\Omega_{1}\right)\right)}+\left\|\partial_{t} \boldsymbol{u}\right\|_{L^{2}\left(0, T ; L^{2}\left(\Omega_{2}\right)^{3}\right)} \\
& \lesssim T^{3 / 2}\left\|\partial_{t} \boldsymbol{j}\right\|_{L^{1}\left(0, T ; L^{2}\left(\Omega_{2}\right)^{3}\right)},
\end{aligned}
$$

which shows the estimate (3.28).

Taking $\epsilon=\frac{1}{2 T}$ and applying the epsilon inequality, we have

$$
\begin{aligned}
\|\boldsymbol{u}\|_{L^{2}\left(0, T ; L^{2}\left(\Omega_{2}\right)^{3}\right)}^{2} & =\int_{0}^{t} \partial_{\tau}\|\boldsymbol{u}(\cdot, \tau)\|_{L^{2}\left(0, T ; L^{2}\left(\Omega_{2}\right)^{3}\right)}^{2} \mathrm{~d} \tau \\
& \leq \frac{1}{2}\|\boldsymbol{u}\|_{L^{2}\left(0, T ; L^{2}\left(\Omega_{2}\right)^{3}\right)}^{2}+2 T^{2}\left\|\partial_{t} \boldsymbol{u}\right\|_{L^{2}\left(0, T ; L^{2}\left(\Omega_{2}\right)^{3}\right)}^{2}
\end{aligned}
$$

It follows from the above inequality and (3.43) that

$$
\|\boldsymbol{u}\|_{L^{2}\left(0, T ; L^{2}\left(\Omega_{2}\right)^{3}\right)}^{2} \lesssim T^{2}\left\|\partial_{t} \boldsymbol{u}\right\|_{L^{2}\left(0, T ; L^{2}\left(\Omega_{2}\right)^{3}\right)}^{2} \lesssim T^{5}\left\|\partial_{t} \boldsymbol{j}\right\|_{L^{1}\left(0, T ; L^{2}\left(\Omega_{2}\right)^{3}\right)}^{2},
$$

which implies the estimate (3.29).

4. Conclusion. In this paper we have studied the time-domain acoustic-elastic interaction problem in an unbounded structure in the three-dimensional space. The problem models the wave propagation in a two-layered medium consisting of the air/fluid and the solid due to an active source located in the solid. We reduce the scattering problem into an initial-boundary value problem by using the exact TBC. We establish the well-posedness and the stability for the variational problem in the $s$-domain. In the time-domain, we show that the reduced problem has a unique weak solution by using the energy method. The main ingredients of the proofs are the Laplace transform, the Lax-Milgram lemma, and the Parseval identity. We also obtain a priori estimates with explicit time dependence for the quantities of acoustic wave pressure and elastic wave displacement by taking special test functions to the time-domain variational problem.

\section{REFERENCES}

[1] I. Abubakar, Scattering of plane elastic waves at rough surfaces. I, Proc. Cambridge Philos. Soc., 58 (1962), pp. 136-157.

[2] T. Arens and T. Hohage, On radiation conditions for rough surface scattering problems, IMA J. Appl. Math., 70(2005), pp. 839-847.

[3] S. N. Chandler-Wilde, E. Heinemeyer, and R. Potthast, Acoustic scattering by mildly rough unbounded surfaces in three dimensions, SIAM J. Appl. Math., 66 (2006), pp. 10021026.

[4] S. N. Chandler-Wilde, And P. Monk, Existence, uniqueness, and variational methods for scattering by unbounded rough surfaces, SIAM J. Math. Anal., 37 (2005), pp. 598-618.

[5] S. N. Chandler-Wilde And B. Zhang, Scattering of electromagnetic waves by rough interfaces and inhomogeneous layers, SIAM J. Math. Anal., 30 (1999), pp. 559-583.

[6] Q. Chen and P. Monk, Discretization of the time domain CFIE for acoustic scattering problems using convolution quadrature, SIAM J. Math. Anal., 46 (2014), pp. 3107-3130.

[7] Z. Chen And J.-C. NÉdÉLEC, On Maxwell's equations with the transparent boundary condition, J. Comput. Math., 26 (2008), pp. 284-296.

[8] A. M. Cohen, Numerical methods for Laplace transform inversion, vol. 5, Numerical Methods and Algorithms, Springer, New York, 2007.

[9] A. G. Dallas, Analysis of a limiting-amplitude problem in acousto-elastic interactions, Technical report, DTIC Document, 1989.

[10] J. Donea, S. Giuliani, and J.-P. Halleux, An arbitrary Lagrangian-Eulerian finite element method for transient dynamic fluid-structure interactions, Comput. Methods Appl. Mech. Engr., 33(1982), pp. 689-723. 
[11] T. M. Elfouhaily and C.-A. GuÉRin, A critical survey of approximate scattering wave theories from random rough surfaces, Waves Random Media, 14(2004), pp. R1-R40.

[12] O. V. Estorff and H. Antes, On fem-bem coupling for fluid-structure interaction analyses in the time domain, Internat. J. Numer. Methods Engrg., 31 (1991), pp. 1151-1168.

[13] B. Flemisch, M. Kaltenbacher, and B. I. Wohlmuth, Elasto-acoustic and acoustic-acoustic coupling on non-matching grids, Internat. J. Numer. Methods Engrg., 67 (2006), pp. 17911810.

[14] J. FoKKEMA, Reflection and transmission of elastic waves by the spatially periodic interface between two solids (theory of the integral-equation method), Wave Motion, 2 (1980), pp. 375-393.

[15] J. Fokkema and P. VAn den Berg, Elastodynamic diffraction by a periodic rough surface (stress-free boundary), J. Acoust. Soc. Amer., 62 (1977), pp. 1095-1101.

[16] Y. Gao And P. Li, Analysis of time-domain scattering by periodic structures, J. Differential Equations, to appear.

[17] H. HAdDAR AND A. Lechleiter, Electromagnetic wave scattering from rough penetrable layers, SIAM J. Math. Anal., 43 (2011), pp. 2418-2443.

[18] M. Hamdi And P. Jean, A mixed functional for the numerical resolution of fluid-structure interaction problems, In Aero-and Hydro-Acoustics, pp. 269-276. Springer, 1986.

[19] G. C. HsiaO, On the boundary-field equation methods for fluid-structure interactions, In Problems and methods in mathematical physics (Chemnitz, 1993), vol. 134, Teubner-Texte Math., pp. 79-88. Teubner, Stuttgart, 1994.

[20] G. C. Hsiao, R. E. Kleinman, and L. S. Schuetz, On variational formulations of boundary value problems for fluid-solid interactions, In Elastic wave propagation (Galway, 1988), vol. 35, North-Holland Ser. Appl. Math. Mech., pp. 321-326, North-Holland, Amsterdam, 1989.

[21] G. Hu, A. KIRSCh, AND T. Yin, Factorization method in inverse interaction problems with biperiodic interfaces between acoustic and elastic waves, Inverse Probl. Imaging, 10 (2016), pp. 103-129.

[22] J.-M. Jin And D. J. Riley, Finite Element Analysis of Antennas and Arrays, John Wiley \& Sons, 2009.

[23] A. Lechleiter and S. Ritterbusch, A variational method for wave scattering from penetrable rough layers, IMA J. Appl. Math., 75 (2010), pp. 366-391.

[24] J. Li And Y. Huang, Time-Domain Finite Element Methods for Maxwell's Equations in Metamaterials, vol. 43, Springer Series in Computational Mathematics, Springer, Heidelberg, 2013.

[25] P. Li And J. Shen, Analysis of the scattering by an unbounded rough surface, Math. Methods Appl. Sci., 35 (2012), pp. 2166-2184.

[26] P. Li, L.-L. Wang, And A. Wood, Analysis of transient electromagentic scattering from a three-dimensional open cavity, SIAM J. Appl. Math., 75 (2015), pp. 1675-1699.

[27] P. Li, H. WU, AND W. ZhenG, Electromagnetic scattering by unbounded rough surfaces, SIAM J. Math. Anal., 43 (2011), pp. 1205-1231.

[28] P. Li, G. Zheng, And W. Zheng, Maxwell's equations in an unbounded structure, Math. Methods Appl. Sci., to appear.

[29] C. J. Luke And P. A. Martin, Fluid-solid interaction: acoustic scattering by a smooth elastic obstacle, SIAM J. Appl. Math., 55 (1995), pp. 904-922.

[30] J. A. OGiLvy, Theory of Wave Scattering from Random Rough Surfaces, Adam Hilger, Ltd., Bristol, 1991.

[31] D. J. Riley and J.-M. Jin, Finite-element time-domain analysis of electrically and magnetically dispersive periodic structures, IEEE Trans. Antennas and Propagation, 56 (2008), pp. 3501-3509.

[32] J. Sherwood, Elastic wave propagation in a semi-infinite solid medium, Proceedings of the Physical Society, 71 (1958), pp. 207.

[33] D. Soares and W. Mansur, Dynamic analysis of fluid-soil-structure interaction problems by the boundary element method, J. Comput. Phys., 219 (2006), pp. 498-512.

[34] F. Trèves, Basic Linear Partial Differential Equations, Pure and Applied Mathematics, vol. 62, Academic Press, New York-London, 1975,

[35] A. G. Voronovich, Non-classical approaches in the theory of wave scattering from rough surfaces, Mathematical and numerical aspects of wave propagation (Golden, CO, 1998), pp. 84-88. SIAM, Philadelphia, PA, 1998.

[36] B. WANG AND L. WANG, On L2-stability analysis of time-domain acoustic scattering problems with exact nonreflecting boundary conditions, J. Math. Study, 1 (2014), pp. 65-84.

[37] L.-L. WANG, B. WANG, AND X. ZhaO, Fast and accurate computation of time-domain acoustic 
scattering problems with exact nonreflecting boundary conditions, SIAM J. Appl. Math., 72 (2012), pp. 1869-1898.

[38] K. F. WARnick And W. C. Chew, Numerical simulation methods for rough surface scattering, Waves Random Media, 11 (2001), pp. R1-R30.

[39] T. Yin, G. Hu, L. Xu, and B. Zhang, Near-field imaging of obstacles with the factorization method: fluid-solid interaction, Inverse Problems, 32 (2016), pp. 015003.

[40] B. Zhang and S. N. Chandler-Wilde, Acoustic scattering by an inhomogeneous layer on a rigid plate, SIAM J. Appl. Math., 58 (1998), pp. 1931-1950. 